Farid A. Badria. / International Journal of Research in Pharmaceutical and Nano Sciences. 9(2), 2020, 46-71.

Research Article

CODEN: IJRPJK

ISSN: $2319-9563$

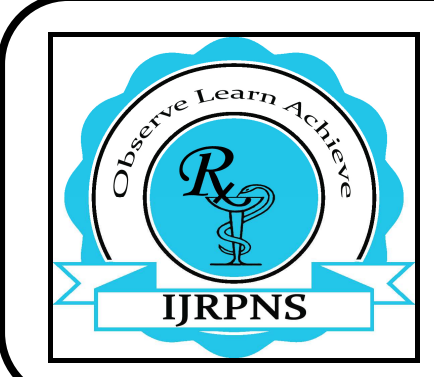

International Journal of Research in

Pharmaceutical and Nano Sciences

Journal homepage: www.ijrpns.com

https://doi.org/10.36673/IJRPNS.2020.v09.i02.A08

\title{
NATURAL PRODUCTTS AND MEDICINAL PLANTS: BOOSTING IMMUNE SYSTEM AGAINST COVID-19 AND OTHER VIRUSES
}

\author{
Farid A. Badria*1 \\ ${ }^{1 *}$ Department of Pharmacognosy, Mansoura University, Faculty of Pharmacy, Mansoura 35516, Egypt.
}

\section{ABSTRACT}

In 2019, the COVID-19 pandemic caused by SARS-CoV-2 in Wuhan, China and raised a worldwide fear with no effective treatment or protection. Therefore, all researchers are racing to provide a solution to this catastrophe either via vaccination or drug development or drug repurposing. Regardless the high fatalities associated with (COVID-19) pandemic, many studies have shown that persons with strong immune systems are more likely not to be infected with the virus or even if they get infected, they have higher chances of surviving the scourge. Until now, several local herbs have been shown to have antibacterial and antiviral properties. Although there are no cures for the flu viruses including coronavirus, researchers suggest many natural remedies can ease the symptoms. They have been shown to provide relief and prevent viral infections. Earlier, The liver research laboratory (FAB-Lab, Faculty of Pharmacy, Mansoura University, Mansoura, Egypt) presented several approaches for a better utilization of natural products as potential antiviral; Anti-herpes (Badria et al, 2003) ${ }^{1}$ and immunomodulators (Mikhaeil et al, 2003) ${ }^{2}$. Here we present a comprehensive review on the chemistry, biology, and therapeutic applications of many natural products as possible therapy and/or prophylactic for many viral diseases including COVID-19.

\section{KEYWORDS}

COVID-19, FAB-Lab, Immunomodulators and Natural Products.

\section{Author for Correspondence:}

Farid A. Badria,

Department of Pharmacognosy,

Mansoura University, Faculty of Pharmacy,

Mansoura 35516, Egypt.

Email: faridbadria@gmail.com

Available online: www.uptodateresearchpublication.com

\section{INTRODUCTION}

The liver research laboratory (FAB-Lab, Faculty of Pharmacy, Mansoura University, Mansoura, Egypt) presented several approaches for a better utilization of natural products as potential therapeutic agents including the following,

- Anti-herpes activity of isolated compounds from frankincense (Badria et al, 2003) ${ }^{3}$.

March - April 46 
Farid A. Badria. / International Journal of Research in Pharmaceutical and Nano Sciences. 9(2), 2020, 46-71.

- Chemistry and immunomodulatory activity of frankincense oil (Mikhaeil et al, 2003) ${ }^{2}$.

- Mirazid: a new schistosomicidal drug (Badria et al, 2001) $)^{4}$.

- Is man helpless against cancer? An environmental approach: antimutagenic agents from Egyptian food and medicinal preparations $(\text { Badria, 1994) })^{5}$.

- Free-B-Ring flavonoids as potential lead compounds for colon cancer therapy (Ibrahim $e t$ $a l, 2014)^{6}$.

- Immune-modulatory potentials of antineoplaston A-10 in breast cancer patients (Badria et al, 2000a) ${ }^{7}$.

- Potential utility of anti-neoplaston A-10 levels in breast cancer (Badria et al, 2000b) ${ }^{8}$.

There are number of examples which deal with enzymes as drug targets involved in the designing of enzyme inhibitors from commonly available natural products, such as;

- Olive and ginkgo extracts as potential cataract therapy with differential inhibitory activity on aldose reductase (Elimam et al, 2017) ${ }^{9}$.

- Flavonoids containing an alpha-keto group as a new type of tyrosinase inhibitors from natural products as potential treatments for hyperpigmentation (Badria, 2001) ${ }^{10}$.

- Cycloartane Glycoside: A New Lactate Dehydrogenase Inhibitor, from the Aerial part of Agriculture Waste of Watermelon (El-attar $e t$ $a l, 2015)^{11}$.

Later, modulation of different biological activities via semi-synthesis of commonly available natural products was extensively studied by Badria's group including the following,

- Betulinic acid analogues as potent topoisomerase inhibitors (Abdel Bar et al, $2009)^{12}$.

- Synthesis, Docking, Cytotoxicity, and LTA4H Inhibitory Activity of New Gingerol Derivatives as Potential Colorectal Cancer Therapy (ElNaggar et al, 2017) ${ }^{13}$.

Available online: www.uptodateresearchpublication.com
- Design and pharmacophore modeling of bi-aryl methyl eugenol analogs as breast cancer invasion inhibitors (Abdel Bar et al, 2010) ${ }^{14}$.

- Approach for chemosensitization of cisplatinresistant ovarian cancer by cucurbitacin B (ElSenduny et al, 2016) ${ }^{15}$.

- Derivatization, molecular docking and in vitro acetyl cholinesterase inhibitory activity of glycyrrhizin as a selective anti-Alzheimer agent (Abdel Bar et al, 2019) ${ }^{16}$.

All of the previous examples are about using one compound that targets single enzyme (the on-target approach), with high selectivity to prevent any undesired effects from mis-targeting the other targets (the off-targets). Previously it was undesirable for drugs to inhibit many targets, because of the adverse effects. Though, the complexity of the current hopeless pathologies has demonstrated clearly that such single-target drugs are insufficient to achieve a therapeutic effect. In parallel, it was found that molecules targeting more than one protein may have a safer profile when compared to the single-targeted (Ramsay et al, 2018) ${ }^{17}$, (Badria and Ahmed, 2018) ${ }^{18}$.

Detection as well as elimination relay on bonding of immune cellular surface receptors as well as chemical binding to pathogens to trigger complex interaction of signaling of immune response.

Innate immune system is a term which describes the part of the immunity which is present since born; that is, it does not acclimatize to particular pathogens (c.f. Adaptive immunity). It is the first line of defense, to give the other immune arm, adaptive immunity, time to construct a precise response. Innate immune system relay primarily on complement "chemical response system", and the phagocytic/ endocytic systems, that involve roaming of scavenger cells, mainly macrophages, which detect / engulf foreign molecules and pathogen, maintain clearance of debris and pathogens.

On the other hand, adaptive immune system consists mainly of specific white blood cells, lymphocytes that circulate throughout the entire body via blood circulation as well as lymph March - April 
Farid A. Badria. / International Journal of Research in Pharmaceutical and Nano Sciences. 9(2), 2020, 46-71.

systems. The name is due to its nature of adaptation or learning how to recognize and detect specific type of pathogen, as well as retaining a memory to speed up faster response on future exposure. The primary response (from which the cells learn) is slow, often becomes apparent after several days of the initial infection, and may take up weeks to clear pathogen. After that, the immune system gain memory (learned) of that specific kind of causative pathogen. If the body, again, got infected by the same pathogen, the adapted immune system remembers it, and so produces more rapid and efficient responses, called secondary responses. This response is fast enough to produce no clinical manifestations. This memory can be temporary or may persist up to life-time.

Cellular immunity (Cell-mediated) is the simple or complex actions, reactions and interactions of many leukocyte activities. This type depends mainly on $\mathrm{T}$ (Thymus) lymphocytes, responsible for the delayed immune response. The T-cells is sensitized by its first exposure to an antigen. Humoral immunity (Antibody-mediated) is the defense mechanisms achieved through circulating antibodies which are secreted by B (Bone cell) lymphocytes. This type of immunity is the antigen-antibody response (Hofmeyr, 2001 ${ }^{19}$, Organ, 2009) ${ }^{20}$.

\section{Organs of the Immune System, (Janeway, et al,} 2005) $)^{21}$

\section{Bone Marrow}

All types of immune cells primarily originate from the bone marrow stem cells through a process of hematopoiesis. These cells either mature or migrate as precursors cells to achieve maturation elsewhere. The bone marrow produces immature thymocytes, B-cells, granulocytes and natural killer in addition to red blood cells and platelets.

\section{Thymus}

Thymus is the organ in which thymocytes (prothymocytes) achieve maturation in a process referred to as "thymic education". Beneficial T cells are spared, while T-cells that may evoke autoimmunity are eliminated. The mature "educated/selected" T-cells are then released into the circulation.

Available online: www.uptodateresearchpublication.com

\section{Spleen}

The spleen is the blood immunologic filter. It consists of macrophages, natural killer cells, dendritic cells, T-cells, B-cells and red blood cells. It captures and filters foreign antigens from the blood present them to effector cells as well as Bcells activated to produce antibodies in large amounts. In spleen, old red blood cells are destroyed.

\section{Lymph nodes}

The lymph nodes also function as filter for the lymph fluid that drains from most of body tissues. Lymph nodes spread throughout the body and consists mainly of $\mathrm{T}$ - and B-cells, macrophages and dendritic cells.

\section{Cells of the Immune System}

Origin and types of immune system cells are illustrated in Figure No.1 and are classified as follows:

\section{T-Cells}

There are two major subsets of T-cells that are functionally and phenotypically different. The Thelper (CD4+), which is immune coordinator, its main function is to potentiate immune responses by secreting specialized cytokines which activate other white blood cells. T-killer/suppressor subset $(\mathrm{CD} 8+)$ is the other type of T-cell which is important in direct killing of viral-infected cells, certain tumor cells, and some parasites as well as down-regulation of over immune responses. T-cells often depend on the lymph nodes and spleen as a site of activation, they also present in other body tissues such as lung, liver, intestinal and reproductive tracts and blood circulation.

\section{B-Cells}

Antibodies production is the major function of Blymphocytes. Antigen-antibody reaction is critical mean for signaling to other cells to kill, engulf or remove that substance from the body.

\section{Natural Killer Cells}

Natural killer cells, (NK-cells), are similar to the killer T-cell subset (CD8+ T-cells) that directly kill certain tumors and viral-infected cells. NK-cells differ from $\mathrm{CD} 8+$ in that they do not need prior lymphoid organ conference before killing.

March - April 
Farid A. Badria. / International Journal of Research in Pharmaceutical and Nano Sciences. 9(2), 2020, 46-71.

Activated NK-cells (by CD4+ cytokines) will produce their action more effectively.

\section{Granulocytes [Polymorph-nuclear (PMN)] Leukocytes}

Granulocytes are divided into three cellular types depending on their dyes' staining characteristics to Basophils, Eosinophils and Neutrophil. These cells remove pathogen from the body via engulfment degradation using specific enzymes.

\section{Macrophages}

Macrophages known as scavengers and antigenpresenting cells (APC) due to their action of picking up and ingestion of foreign materials then present them to other immune system such as B-cells and T-cells. Macrophages upon stimulation exhibit increased phagocytosis and also secretions.

\section{Dendritic cells}

Unfortunately, there is little information about dendritic cells as they are hard to be isolated. Recently, dendritic cells were found to bind high amount of AIDS virus, and may act as a reservoir that is during an activation event is transmitted to CD4+ T cells.

Cytokines, category of small proteins, are of major importance in cell signaling. Cytokines comprise interferons, interleukins, chemokines, lymphokines and tumor necrosis factor but generally not hormones nor growth factors. They are remarkably important in the immune system; they regulate the growth, maturation, and responsiveness of specific cell populations and harmonize between humoral and cell-based immune responses. Cytokines are released by special cells as macrophages, $\mathrm{T}$ lymphocytes B-lymphocytes, and mast cells, as well as fibroblasts, endothelial cells, and various stromal cells. They either affect the behavior of other cells, or even the releasing cell itself. More than one type of cell can produce the same cytokine to act through receptors. Also, cytokines may inhibit or enhance other cytokines' action in complicated manners.

Milk thistle has been proven to increase humoral and cellular activity (Wilasrusmee et al, 2002) ${ }^{22}$. Ginseng enhances production of macrophages, B and T cells (Liou et al, 2004) ${ }^{23}$. Echinacea is being tested as an immune stimulant (Melchart et al, 1994) $)^{24}$.

Because of cytokines importance in the immune system, their modulation by natural products may produce crucial effects on immunity of the host leading to enhancement of its defensive mechanisms. Figure No.2.

Understanding plant based drug immunological profile is key of interest. The effect may be antagonistic, since administration of high doses of an agent tends to suppress the immunity while the same in low doses might become immunostimulant. Also, in some cases the agent may be immunosuppressive and in other case it tends to be immunostimulatory. (Billiau and Matthys, 2001) ${ }^{25}$. Immunomodulator can be used as an alternative to conventional chemotherapy for a variety of diseases especially under the conditions of immuneimpairment. Medicinal plants are a rich source of molecules that are claimed to induce nonspecific immunomodulation. To regulate the normal immunological function, both stimulation and suppression need to be regulated so to normalize patho-physiological processes, hence the name 'immunomodulatory agents'. It is important to note that, there are limitations to the use of such agents, due to increased risk of infection as well as generalized or uncontrolled effect throughout the immune system. (Killestein et al, 2003 ${ }^{26}$, Ordway et $a l, 2003)^{27}$.

Due to toxicity, low efficacy and elevated cost of synthetic agents, the search for more effective and safer natural immunomodulators is becoming a field of major interest all over the world.

Several medicinal plant extracts used in traditional medicines have shown to be immunomodulators. Some of medicinal plants that exhibit immunomodulatory activities are presented in Table No.1.

\section{Immunomodulatory natural products}

Several molecules with immunomodulatory action have been also reported. Various secondary metabolites (e.g., alkaloids, glycosides, saponins, flavonoids, coumarins and sterols) exhibit a wide

March - April 
Farid A. Badria. / International Journal of Research in Pharmaceutical and Nano Sciences. 9(2), 2020, 46-71.

range of immunomodulating activity, (Ganju et al, $2003)^{67}$.

\section{Glycosides}

Glycosides are plant or animal derived organic compounds that upon enzymatic or acid hydrolysis, yield one or more sugar moieties. There are number of glycosides that have immunomodulatory activities; iridoids and anthraquinone glycosides (Mehrotra et al, 2002) ${ }^{97}$ as shown in Figure No.3.

\section{Flavonoids}

Many flavonoids retains immunomodulatory effect; e.g. eupalitin and centaureidin (Davis and Kuttan, $2000)^{98}$, (Bhattacharya et al, 2000) ${ }^{99}$. Figure No.4.

\section{Coumarins}

Coumarinsare benzo- $\alpha$-pyrone derivatives (Makare et al, 2001) ${ }^{100}$. Some coumarins exert immunomodulation effects. Example 6, 7dihydroxycoumarin (Esculetin) which is a coumarin derivative isolated from many medicinal plants such as Citrus limonia, Artemisia capillaries, and Euphorbia lathyris. It is known to exhibit other diverse biological activities including free radical scavenging, tyrosinase-inhibition, lipoxygenaseinhibitory activity, suppressive activity on oxidative damage to DNA, and cancer chemo-prevention / antitumor activities (Leung et al, 2005) ${ }^{101}$, Figures No.5.

\section{Alkaloids}

Alkaloids are organic nitrogenous compounds that have alkali like behavior (basic nature), hence the name, normally heterocyclic, limited in distribution and have special and wide range of effects on body physiological functions including immunomodulatory activities (Leung et al, 2005) ${ }^{101}$. As an example, Murraya koenigii, Achillea millefolium, Actinidia macrosperma and Cissampelos pareira, Figure No.6.

Thiosulfinates are organic sulfur compounds containing the linkage R-S (O)-S-R. They exert potent immunomodulatory and adaptogenic properties, e.g., Allicinthiosulfinates derived from Allium hirtifolium (Jafarian et al, 2010) ${ }^{102}$. Figure No.7.

\section{Polysaccharides}

Polysaccharides from botanical sources exhibit versatile beneficial therapeutic properties, it is believed that these effects are due to the modulation of innate immunity especially macrophage function. So, the assay and testing of plant polysaccharides provides a special approach to discover novel adjuvants and therapeutic agents that produce desired immunomodulation. The enhancing effect of polysaccharide on lymphocyte proliferation was correlated to its effect on intracellular calcium ions $\left(\mathrm{Ca}^{2+}\right)$ delivery. Salicornia herbacea extract showed to be able to activate monocytic cells and induced its differentiation into macrophages (Alamgir and Uddin, 2010) ${ }^{103}$ (Rios, 2010) ${ }^{104}$, Figure No.8.

Volatile oils, monoterpenes and sesquiterpenes

Terpenes and terpenoids are mainly volatile oils (Rios, 2010) ${ }^{104}$. A lot of volatile oils exhibit immunomodulatory activity, e.g., eugenol from Ocimum sanctum, Figure No.9.

\section{Diterpenes}

The immunomodulatory activity of andrographolide, a diterpene isolated from Andrographis paniculata has been also reported, Figure No.9.

\section{Saponins and triterpenes}

Triterpenoidal saponins may produce immunomodulatory effects, such as those isolated from Glycyrrhiza glabra and Boswellia spp. (Badria et al, 2003) ${ }^{1}$, Figure No.10.

\section{Semisynthetic glycyrrhetinic acid derivatives}

Semisynthetic modifications are done either to improve potency, enhance pharmacokinetics or to change pharmacodynamics of a lead molecule.

Glycyrrhizin is a triterpenoidsaponin glycoside that contains two molecules of glucuronic acid as glyconepart. Its derivatization may lead to enhancement of its already existing activities or development of newer ones. Glycyrrhetinic acid comprises three main functionalities which can be considered as a main target of simple modifications, 3-hydroxyl group that is involved in glycoside formation, 11- $\alpha, \beta$-unsaturatedketone group and 30carboxylic group.

March - April 50 
Farid A. Badria. / International Journal of Research in Pharmaceutical and Nano Sciences. 9(2), 2020, 46-71.

Several modifications have been done which will be discussed below under Figure No.11, to review the modification and its significance on the main derivative of glycyrrhizin, glycyrrhetinic acid "the aglycone of glycyrrhizin".

Modifications made on position 3-hydroxy ( $\mathbf{R}_{1}$ A ring)

\section{Hydroxylation}

Addition of two hydroxyl groups at positions C-1 and $\mathrm{C}-2$ produced trihydroxylated derivatives which showed markedly enhanced antibacterial activity against $G$ +vestrains even more potent than the positive controls (Huang et al, 2016) ${ }^{105}$.

\section{Acylation}

Introduction of acetyl group using acetylating agents as acetic anhydride produced chemical protection of hydroxyl group, and increased lipophilicity of the molecule (Terasawa et al, 1992) ${ }^{106}$. 3-Acetyl-18 $\beta$-glycyrrhetinic acid showed significant curative effect for the treatment of gastric and duodenal ulcer (Zhang et al, 2012) ${ }^{107}$.

Acylation with di-carboxylic acid as succinic acid affected promotion of anti-tumor activity (Terasawa et al, 1992) ${ }^{106}$.

\section{Oxidation}

Formation of 3-oxo moiety slightly increased lipophilicity which intern produced higher antitumor-promoter activity. (Terasawa et al, 1992) ${ }^{106}$.

Modifications made on position 30-carboxyllic acid $\left(\mathbf{R}_{2}\right)$

\section{Esterification}

Methyl glycyrrhetinate has been synthesized by hydrothermal method with glycyrrhizic acid as a precursor. The main purpose of such methylation is protection of carboxylic acid moiety and increase hydrophobic character of the compound. (Zhang et al, 2010) ${ }^{108}$.

Glycyrrhizin penta- $O$-nicotinate "Niglizin" inhibited HIV-1 and HIV-2 in the model of the chronic infection in vitro (Baltina, 2003) ${ }^{109}$.
The anti-inflammatory activity of penta- $O$-(4-nitro) benzoate and penta- $O$-cinnamate was more pronounced in the carrageenan and formaldehyde induced models of inflammation in mice (Baltina, $2003)^{109}$.

\section{Amidation}

Glycyrrhizin containing cysteine-moiety of "Cys(Bzl) and Cys(Bzl)-Val" residues attached to the glycone part of the molecule stimulated the primary response of immune system and the reaction of mice delayed-type hypersensitivity. (Kondratenko et al, 2004) ${ }^{110}$.

Glycyrrhizin heterocyclic amide and ureids were more potent and less toxic anti-inflammatory agents in comparison to prednisolone in the mice inflammation induced by formaldehyde and carrageenan (Baltina, 2003) ${ }^{109}$.

Glycopeptide and dipeptide derivatives of glycyrrhizin, methyl esters of amino acids (AlaOMe, LeuOMe, GluOMe- OMe), were found immunomodulators that stimulated the primary immune response in mice in the model of delayedtype hypersensitivity (Baltina, 2003) ${ }^{109}$.

Modifications made on position 11$\alpha, \beta$ unsaturated-ketone $\left(\mathbf{R}_{3}\right)$

11-Oxo group is the chief reason for the apoptotic effect of glycyrrhetinic acid and its derivatives (Csuk et al, 2011) ${ }^{111}$.

11-Deoxoglycyrrhetinic acid and its salt were used as immune modulators, anti-inflammatory agentsand antiulcer agents (Nakashima et al, 1987) ${ }^{112}$.

11-Deoxyglycyrrhetinic amide and 3-amino-11deoxyglycyrrhetinic acid exhibited significant effects on treatment of ulcers, and antiinflammatory against aseptic arthritis of various animals (Zhang et al, 2012) ${ }^{107}$. 
Farid A. Badria. / International Journal of Research in Pharmaceutical and Nano Sciences. 9(2), 2020, 46-71.

Table No.1: Some common plant-derived immunomodulators

\begin{tabular}{|c|c|c|c|c|c|}
\hline S.No & Botanical name & Part used & Active constituents & $\begin{array}{c}\text { Other biological } \\
\text { activities }\end{array}$ & Ref. \\
\hline 1 & $\begin{array}{l}\text { Achillea millefolium } \\
\text { (Asteraceae) }\end{array}$ & Leaves & $\begin{array}{l}\text { Flavonoids, alkaloids, } \\
\text { coumarins, triterpenes }\end{array}$ & $\begin{array}{l}\text { Anti-inflammatory, } \\
\text { antispasmodic, } \\
\text { antipyretic, diuretic. }\end{array}$ & $\begin{array}{c}\text { (Sharififar et al, } \\
2009)^{28}\end{array}$ \\
\hline 2 & Aloe vera Tourn (Liliaceae) & $\begin{array}{l}\text { Gel from } \\
\text { leaves }\end{array}$ & $\begin{array}{l}\text { Anthraquinone } \\
\text { glycosides }\end{array}$ & $\begin{array}{c}\text { Purgative, } \\
\text { emmenagogue, } \\
\text { emollient, } \\
\text { ant-inflammatory. }\end{array}$ & $\begin{array}{c}\text { (Hamman, } 2008^{29}, \\
\text { Sharififar et al, } \\
\text { 2009) }\end{array}$ \\
\hline 3 & $\begin{array}{c}\text { Andrographis paniculata } \\
\text { (Acanthaceae) }\end{array}$ & Leaves & Diterpenes & $\begin{array}{l}\text { Hepatoprotective, } \\
\text { antispasmodic, } \\
\text { blood purifier, } \\
\text { febrifuge. }\end{array}$ & $\begin{array}{l}\text { (Varma et al, } \\
2011)^{30}\end{array}$ \\
\hline 4 & $\begin{array}{c}\text { Abutilon indicum } \\
\text { (Malvaceae) }\end{array}$ & Whole plant & Flavonoids, triterpenoids & $\begin{array}{c}\text { Diuretic, } \\
\text { antibacterial. }\end{array}$ & $\begin{array}{c}\text { (Dashputre and } \\
\text { Naikwade, 2010) }\end{array}$ \\
\hline 5 & $\begin{array}{c}\text { Asparagus racemosus } \\
\text { (Liliaceae) }\end{array}$ & Roots & Saponins, sitosterols & $\begin{array}{l}\text { Ulcer healing agent, } \\
\text { nervine tonic, } \\
\text { anti-gout. }\end{array}$ & $\begin{array}{c}\text { (Bopana and } \\
\text { Saxena, 2007) }\end{array}$ \\
\hline 6 & $\begin{array}{c}\text { Allium hirtifolium } \\
\text { (Alliaceae) }\end{array}$ & Herb & $\begin{array}{c}\text { Thiosulfinates, } \\
\text { flavonoids }\end{array}$ & $\begin{array}{c}\text { Anti-rheumatic, } \\
\text { anti-inflammatory. }\end{array}$ & $\begin{array}{c}\text { (Dashputre and } \\
\text { Naikwade, 2010) }\end{array}$ \\
\hline 7 & $\begin{array}{c}\text { Alternanthera tenella } \\
\text { (Amaranthaceae) }\end{array}$ & Herb & Flavonoids, triterpenes & $\begin{array}{l}\text { Antitumor, anti- } \\
\text { inflammatory. }\end{array}$ & $\begin{array}{c}\text { (Guerra et al, } \\
2003)^{33}\end{array}$ \\
\hline 8 & $\begin{array}{c}\text { Actinidia macrosperma } \\
\text { (Actinidiaceae) }\end{array}$ & Fruits & Alkaloids, saponins & Anti-leprotic. & $(\mathrm{Lu} \text { et al, 2007) })^{34}$ \\
\hline 9 & $\begin{array}{r}\text { Acacia catechu } \\
\text { (Leguminosae) }\end{array}$ & Leaf & Flavonoids, quercetin & $\begin{array}{l}\text { Hypoglycaemic, } \\
\text { astringent. }\end{array}$ & $\begin{array}{c}\text { (Ismail and Asad, } \\
2009)^{35}\end{array}$ \\
\hline 10 & $\begin{array}{c}\text { Acanthopanax sessiliflorus } \\
\text { (Araliaceae) }\end{array}$ & $\begin{array}{l}\text { Shoots and } \\
\text { roots }\end{array}$ & Biopolymers & $\begin{array}{l}\text { Lympho- } \\
\text { proliferative } \\
\text { activity. }\end{array}$ & $\begin{array}{l}\text { (Jeong et al, } \\
2006)^{36}\end{array}$ \\
\hline 11 & $\begin{array}{l}\text { Agelas mauritianus } \\
\text { (Porifera) }\end{array}$ & Sponge & Glycolipid & $\begin{array}{c}\text { Phagocytotic } \\
\text { activity. }\end{array}$ & $\begin{array}{c}\text { (Hung et al, } \\
2007)^{37}\end{array}$ \\
\hline 12 & $\begin{array}{c}\text { Aphanothece halophytica } \\
\text { (Chroococcales) }\end{array}$ & $\begin{array}{c}\text { Cyano } \\
\text { bacterium }\end{array}$ & Exopolysaccharide & $\begin{array}{l}\text { Inhibits influenza } \\
\text { virus. }\end{array}$ & $\begin{array}{l}\text { (Zheng et al, } \\
2006)^{38}\end{array}$ \\
\hline 13 & $\begin{array}{l}\text { Apium graveolens } \\
\text { (Apiaceae) }\end{array}$ & Leaves, seeds & Flavonoids, coumarins & Anti-inflammatory. & $\begin{array}{c}\text { (Cherng et al, } \\
2008)^{39}\end{array}$ \\
\hline 14 & Ardisia spp. (Myrsinaceae) & $\begin{array}{c}\text { Shrub, } \\
\text { Branches and } \\
\text { leaves } \\
\end{array}$ & $\begin{array}{l}\text { Peptides, saponins, } \\
\text { Isocoumarins, quinones } \\
\text { and alkyl phenols }\end{array}$ & $\begin{array}{c}\text { Anti-metastatic } \\
\text { drug, anti-HIV } \\
\text { property. }\end{array}$ & $\begin{array}{l}\text { (Kobayashi and De } \\
\text { Mejia, 2005) }\end{array}$ \\
\hline 15 & $\begin{array}{l}\text { Aristolochia spp. } \\
\text { (Aristolochiaceae) }\end{array}$ & Leaves & Aristolochic acid & $\begin{array}{l}\text { Antiangiogenic, } \\
\text { employed in prostate } \\
\text { cancer. }\end{array}$ & $\begin{array}{l}\text { (Wang et al, } \\
2010)^{41}\end{array}$ \\
\hline
\end{tabular}


Farid A. Badria. / International Journal of Research in Pharmaceutical and Nano Sciences. 9(2), 2020, 46-71.

\begin{tabular}{|c|c|c|c|c|c|}
\hline 16 & $\begin{array}{l}\text { Artemisia anпua } \\
(\text { Compositea })\end{array}$ & Herb & Artemisinin & Immunosuppressive & $\begin{array}{l}\text { (Noori et al, } \\
2004)^{42}\end{array}$ \\
\hline 17 & $\begin{array}{l}\text { Aspergillus spp. } \\
\text { (Trichocomaceae) }\end{array}$ & Fungus & Polyene, triazole & Anti-fungals. & $\begin{array}{l}\text { (Steinbach and } \\
\text { Stevens, 2003) }{ }^{43}\end{array}$ \\
\hline 18 & Boswellia spp. (Burseraceae) & Gum resin & Triterpenes (ursanes) & Hypoglycaemic. & $\begin{array}{c}\text { (Mikhaeil et al } \\
2003)^{2}\end{array}$ \\
\hline 19 & $\begin{array}{l}\text { Bauhinia variegate } \\
\text { (Caesalpiniaceae) }\end{array}$ & $\begin{array}{l}\text { Roots, bark, } \\
\text { buds }\end{array}$ & $\begin{array}{l}\text { Flavonoids, beta- } \\
\text { sitosterol, lupeol }\end{array}$ & $\begin{array}{l}\text { Anti-fungal, } \\
\text { astringent. }\end{array}$ & $\begin{array}{c}\text { (Ghaisas et al, } \\
2009)^{44}\end{array}$ \\
\hline 20 & $\begin{array}{l}\text { Botryllus schlosseri } \\
\quad \text { (Botryllidae) }\end{array}$ & Tunicates & Cytokines & $\begin{array}{c}\text { Anti-oxidant, } \\
\text { anti-viral, } \\
\text { anti-microbial and } \\
\text { anti-tumoral. }\end{array}$ & $(\text { Ballarin, 2008) })^{45}$ \\
\hline 21 & Bidens pilosa (Asteraceae) & $\begin{array}{l}\text { Flowers, } \\
\text { leaves }\end{array}$ & Polyacetylenes & $\begin{array}{c}\text { Anti-inflammatory, } \\
\text { immunosuppressive, } \\
\text { antibacterial and } \\
\text { antimalarial. }\end{array}$ & $\begin{array}{l}\text { (Chang et al, } \\
\text { 2007) }\end{array}$ \\
\hline 22 & $\begin{array}{l}\text { Boerhaavia diffusa } \\
\text { (Nyctaginaceae) }\end{array}$ & Herb & Alkaloid & Immunostimulatory. & $\begin{array}{l}\text { (Mungantiwar } \\
\text { et al, 1999) }\end{array}$ \\
\hline 23 & Bugula neritina (Bugulidae) & $\begin{array}{c}\text { Marine } \\
\text { invertebrates }\end{array}$ & Macrocyclic lactones & Immunomodulator. & $\begin{array}{l}\text { (Sredni et al, } \\
1990)^{48}\end{array}$ \\
\hline 24 & $\begin{array}{l}\text { Byrsonima crassa } \\
\text { (Malpighiaceae) }\end{array}$ & Leaves & $\begin{array}{c}\text { Flavonoids, tannins, } \\
\text { terpenes }\end{array}$ & $\begin{array}{l}\text { Antimicrobial, } \\
\text { antioxidant. }\end{array}$ & $\begin{array}{c}\text { (Johnston et al, } \\
2009)^{49}\end{array}$ \\
\hline 25 & $\begin{array}{l}\text { Cissampelos pareira } \\
\text { (Menispermiaceae) }\end{array}$ & Roots & Hayatine alkaloids & $\begin{array}{c}\text { Anti-pyretic, } \\
\text { analgesic, anti-lithic. }\end{array}$ & $\begin{array}{c}\text { (Bafna and Mishra, } \\
2010)^{50}\end{array}$ \\
\hline 26 & $\begin{array}{c}\text { Cleome gynandra } \\
\text { (Capperdiceae) }\end{array}$ & $\begin{array}{l}\text { Leaf, seeds, } \\
\text { rots }\end{array}$ & Hexacosanol, kaempferol & Anti-inflammatory. & $\begin{array}{l}(\text { Gaur } \text { et al, } \\
2009)^{51}\end{array}$ \\
\hline 27 & $\begin{array}{c}\text { Chlorophytum borivilianum } \\
\text { (Liliaceae) }\end{array}$ & Roots & Sapogenins & Anti-fungal. & $\begin{array}{l}\text { (Thakur et al, } \\
2007)^{52}\end{array}$ \\
\hline 28 & $\begin{array}{c}\text { Citrus natsudaidai } \\
\text { (Rutaceae) }\end{array}$ & Fruits & Auraptene, flavonoids & Anti-oxidant. & $\begin{array}{l}\text { (Tanaka et al, } \\
1999)^{53}\end{array}$ \\
\hline 29 & $\begin{array}{l}\text { Calendula Officinalis } \\
\text { (Asteraceae) }\end{array}$ & Flowers & $\begin{array}{c}\text { Polysaccharides, } \\
\text { carotenoids, flavonoids, } \\
\text { triterpenoids } \\
\end{array}$ & $\begin{array}{c}\text { Anti-tumor, } \\
\text { anti-viral activity, } \\
\text { anti-HIV properties. }\end{array}$ & $\begin{array}{c}\text { (Mani and } \\
\text { Lawson, 2006) }\end{array}$ \\
\hline 30 & $\begin{array}{l}\text { Camellia sinensis } \\
\text { (Theaceae) }\end{array}$ & Leaves & $\begin{array}{l}\text { Epigallocatechingallate, } \\
\text { quercetin, gallicacid }\end{array}$ & $\begin{array}{l}\text { Anti-cancer activity, } \\
\text { hepatoprotective and } \\
\text { antioxidant. }\end{array}$ & $\begin{array}{l}\text { (Bhatt et al, } \\
2010)^{55}\end{array}$ \\
\hline 31 & $\begin{array}{c}\text { Cannabis sativa } \\
\text { (Cannabaceae) }\end{array}$ & Leaves & Cannabinoids & Immunomodulatory. & $\begin{array}{l}\text { (Killestein et al, } \\
2003)^{26}\end{array}$ \\
\hline 32 & $\begin{array}{l}\text { Carpobrotus edulis } \\
\text { (Aizoaceae) }\end{array}$ & Flowers, fruit & Alkaloids & Immunomodulator. & $\begin{array}{c}(\text { Ordway et al } \\
2003)^{27}\end{array}$ \\
\hline 33 & $\begin{array}{c}\text { Centella asiatica } \\
\text { (Umbelliferae) }\end{array}$ & Herb & Triterpenoidsaponins & Immunomodulator. & $\begin{array}{c}\text { (Mali and } \\
\text { Hatapakki, 2008) }\end{array}$ \\
\hline 34 & $\begin{array}{c}\text { Cordia superba } \\
\text { C. rufescens (Boraginaceae) }\end{array}$ & $\begin{array}{l}\text { Leaf, fruit, } \\
\text { bark }\end{array}$ & Alpha-amyrin & $\begin{array}{l}\text { Anti-inflammatory, } \\
\text { anti-microbial. }\end{array}$ & $\begin{array}{c}(\text { Costa } \text { et al, } \\
2008)^{57}\end{array}$ \\
\hline 35 & $\begin{array}{c}\text { Cistanche deserticola } \\
\text { (Orobanchaceae) }\end{array}$ & Herb & Polysaccharide & $\begin{array}{l}\text { Immunomodulator, } \\
\text { mitogenic and } \\
\text { comitogenic } \\
\text { activities. }\end{array}$ & $\begin{array}{c}\text { (Ebringerova et al, } \\
2002)^{58}\end{array}$ \\
\hline
\end{tabular}

Available online: www.uptodateresearchpublication.com March - April 
Farid A. Badria. / International Journal of Research in Pharmaceutical and Nano Sciences. 9(2), 2020, 46-71.

\begin{tabular}{|c|c|c|c|c|c|}
\hline 36 & Cliona celata (Clionaidae) & Sponge & $\begin{array}{c}\text { Clionamide, } \\
\text { dehydrodopamine }\end{array}$ & $\begin{array}{l}\text { Anti-bacterial } \\
\text { activity. }\end{array}$ & $\begin{array}{l}\text { (Sugumaran and } \\
\text { Robinson, 2010) }\end{array}$ \\
\hline 37 & $\begin{array}{c}\text { Cordyceps militaris } \\
\text { (Clavicipitaceae) }\end{array}$ & Fungus & $\begin{array}{c}\text { Cordycepin, cordyceps } \\
\text { acid }\end{array}$ & Anti-inflammatory. & $(\text { Hsu et al, 2008) })^{60}$ \\
\hline 38 & $\begin{array}{c}\text { Couroupita guianensis } \\
\text { (Lecythidaceae) }\end{array}$ & $\begin{array}{l}\text { Fruits, } \\
\text { flowers }\end{array}$ & $\begin{array}{c}\text { Steroids, flavonoids, } \\
\text { phenolics }\end{array}$ & Anti-fungal. & $\begin{array}{l}\text { (Pradhan et al, } \\
2009)^{61}\end{array}$ \\
\hline 39 & $\begin{array}{l}\text { Crinum latifolium } \\
\text { (Amaryllidaceae) }\end{array}$ & Herb & Alkaloids & Immunomodulator. & $\begin{array}{l}\text { (Zvetkova et al, } \\
2001)^{62}\end{array}$ \\
\hline 40 & $\begin{array}{c}\text { Dracocephalum spp. } \\
\text { (Lamiaceae) }\end{array}$ & Herb & Essential oil & Immunomodulator. & $\begin{array}{c}\text { (Amirghofran } e t \\
a l, 2000)^{63}\end{array}$ \\
\hline 41 & $\begin{array}{c}\text { Echinacea angustifolia } \\
\text { (Asteraceae) }\end{array}$ & Flowers & Polysaccharide & $\begin{array}{l}\text { Treatment for } \\
\text { common cold, } \\
\text { immunomodulator. }\end{array}$ & $\begin{array}{l}\text { (Senchina et al } \\
2005)^{64}\end{array}$ \\
\hline 42 & Eclipta alba (Asteraceae) & Leaves & Triterpenoidglucoside & $\begin{array}{c}\text { Anti-cancer, } \\
\text { anti-leprotic, } \\
\text { analgesic, } \\
\text { anti-oxidant, } \\
\text { anti-myotoxic. }\end{array}$ & $\begin{array}{l}\text { (Jayathirtha and } \\
\text { Mishra, 2004) }\end{array}$ \\
\hline 43 & $\begin{array}{l}\text { Euphorbia hirta } \\
\text { (Euphorbiaceae) }\end{array}$ & Herb & $\begin{array}{l}\text { Quercitol, myricitrin, } \\
\text { gallic acid }\end{array}$ & $\begin{array}{l}\text { Anti-inflammatory } \\
\text { activity, sedative } \\
\text { and anxiolytic } \\
\text { activity. }\end{array}$ & $(\mathrm{SM}, 2009)^{66}$ \\
\hline 44 & $\begin{array}{c}\text { Evolvulus alsinoides } \\
\text { (Convolvulaceae) }\end{array}$ & Herb & Alkaloids & Brain tonic. & $\begin{array}{c}\text { (Ganju et al, } \\
2003)^{67}\end{array}$ \\
\hline 45 & $\begin{array}{l}\text { Ganoderma lucidum } \\
\text { (Polyporaceae) }\end{array}$ & Whole plant & Flavonoids, triterpenes & Antioxidant. & $\begin{array}{c}\text { (Habijanic et al, } \\
2001)^{68}\end{array}$ \\
\hline 46 & $\begin{array}{c}\text { Gymnema sylvestre } \\
\text { (Asclepiadaceae) }\end{array}$ & Leaves & Sapogenins & $\begin{array}{c}\text { Antidiabetic, } \\
\text { diuretic, antibilious. }\end{array}$ & $\begin{array}{l}\text { (Malik et al, } \\
2009)^{69}\end{array}$ \\
\hline 47 & $\begin{array}{c}\text { Haussknechtia elymatica } \\
\text { (Apioideae) }\end{array}$ & Herb & Phenolics & Immunomodulator. & $\begin{array}{c}\text { (Amirghofran } e t \\
a l, 2007)^{70}\end{array}$ \\
\hline 48 & $\begin{array}{c}\text { Heracleum persicum } \\
\text { (Apiaceae) }\end{array}$ & Shurb & $\begin{array}{c}\text { Flavonoids, } \\
\text { furanocoumarins }\end{array}$ & Anti-microbial. & $\begin{array}{c}\text { (Sharififar } \text { et al, } \\
2010)^{71}\end{array}$ \\
\hline 49 & $\begin{array}{c}\text { Hibiscus rosasinensis } \\
\text { (Malvaceae) }\end{array}$ & Flowers & Cyclopropanoids & $\begin{array}{l}\text { Anti-diarrheal, anti- } \\
\text { inflammatory. }\end{array}$ & $\begin{array}{c}(\text { Gaur } \text { et al, } \\
2009)^{51}\end{array}$ \\
\hline 50 & $\begin{array}{c}\text { Hyptis suaveolens } \\
\text { (Lamiaceae) }\end{array}$ & Leaf, flowers & Lupeol, beta-sitosterol & $\begin{array}{c}\text { Carminative, } \\
\text { anti-spasmodic. }\end{array}$ & $(\text { Jain } \text { et al, 2005) })^{72}$ \\
\hline 51 & $\begin{array}{c}\text { Inonotus obliquus } \\
\text { (Hymenochaetaceae) }\end{array}$ & Mushroom & Polysaccharide & Anti-tumor. & $\begin{array}{c}\text { (Caifa Chen et al, } \\
\text { 2007) } \\
\end{array}$ \\
\hline 52 & $\begin{array}{l}\text { Larrea divaricate } \\
\text { (Zygophyllaceae) }\end{array}$ & Herb & Lignans & Anti-inflammatory. & $\begin{array}{c}\text { (Davicino et al } \\
2007)^{74}\end{array}$ \\
\hline 53 & $\begin{array}{l}\text { Lycium barbarum } \\
\text { (Solanaceae) }\end{array}$ & Fruits & $\begin{array}{l}\text { Polysaccharide-protein } \\
\text { complexes }\end{array}$ & Antioxidant. & $(\text { Gan } \text { et al, 2003) })^{75}$ \\
\hline 54 & $\begin{array}{c}\text { Lagenaria siceraria } \\
\text { (Cucurbitaceae) }\end{array}$ & Leaves, fruit & $\begin{array}{c}\text { Cucurbitacin, beta- } \\
\text { glycosidase }\end{array}$ & Purgative, emetic. & $\begin{array}{c}\text { (Deshpande et al, } \\
2008)^{76}\end{array}$ \\
\hline 55 & $\begin{array}{l}\text { Matricaria chamomilla } \\
\text { (Rhabdoviridae) }\end{array}$ & Flowers & Protein & Immunomodulator. & $\begin{array}{c}\text { (De Souza Reis et } \\
a l, 2008)^{77}\end{array}$ \\
\hline 56 & $\begin{array}{c}\text { Mollugo verticillata } \\
\text { (Molluginaceae) }\end{array}$ & Herb & $\begin{array}{l}\text { Quercetin, triterpenoid } \\
\text { glycosides }\end{array}$ & Immunomodulator. & $\begin{array}{c}\text { (Ferreira et al, } \\
2003)^{78}\end{array}$ \\
\hline
\end{tabular}

Available online: www.uptodateresearchpublication.com March - April 
Farid A. Badria. / International Journal of Research in Pharmaceutical and Nano Sciences. 9(2), 2020, 46-71.

\begin{tabular}{|c|c|c|c|c|c|}
\hline 57 & Morus alba (Moraceae) & $\begin{array}{l}\text { Fruits, leaves, } \\
\text { bark }\end{array}$ & Flavonoids, anthocyanins & $\begin{array}{c}\text { Expectorant, hypo- } \\
\text { cholesterolaemic, } \\
\text { diuretic. }\end{array}$ & $\begin{array}{c}\text { (Bharani et al, } \\
\text { 2010) }\end{array}$ \\
\hline 58 & $\begin{array}{c}\text { Moringa oleifera } \\
\text { (Moringaceae) }\end{array}$ & Leaves & $\begin{array}{c}\text { Vitamin A, B, C, } \\
\text { carotenoids, saponins }\end{array}$ & Anti-oxidant. & $\begin{array}{c}\text { (Gupta et al, } \\
2010)^{80}\end{array}$ \\
\hline 59 & Murraya koenigii (Rutaceae) & Leaves & $\begin{array}{l}\text { Coumarin, carbazole } \\
\text { alkaloids, glucoside }\end{array}$ & $\begin{array}{l}\text { Anti-fungal, } \\
\text { insecticidal, } \\
\text { insecticidal. }\end{array}$ & $\begin{array}{l}(\text { Shah } \text { et al } \\
2008)^{81}\end{array}$ \\
\hline 60 & $\begin{array}{c}\text { Nyctanthes arbor-tristis } \\
\text { (Oleaceae) }\end{array}$ & Leaf, seeds & Iridoidglucosides & $\begin{array}{l}\text { Anti-inflammatory, } \\
\text { antispasmodic. }\end{array}$ & $\begin{array}{c}(\text { Kannan et al, } \\
2007)^{82}\end{array}$ \\
\hline 61 & $\begin{array}{l}\text { Ocimum sanctum } \\
\quad \text { (Labiateae) }\end{array}$ & Entire plant & Essential oils & $\begin{array}{c}\text { Stomachic, } \\
\text { anti-spasmodic, } \\
\text { anti-asthmatic, } \\
\text { hepatoprotective. }\end{array}$ & $\begin{array}{c}\text { (Vaghasiya et al, } \\
2010)^{83}\end{array}$ \\
\hline 62 & $\begin{array}{c}\text { Pestalotiopsis leucothes } \\
\text { (Amphisphaeriaceae) }\end{array}$ & Fungus & Terpenes & Immunomodulator. & $\begin{array}{c}\text { (Kumar et al, } \\
2005)^{84}\end{array}$ \\
\hline 63 & $\begin{array}{c}\text { Picrorhiza scrophulariiflora } \\
\text { (Scrophulariaceae) }\end{array}$ & Roots & $\begin{array}{l}\text { Iridoid glycosides, } \\
\text { amphicoside }\end{array}$ & Anti-oxidant. & $(\text { Smit, 2001) })^{85}$ \\
\hline 64 & Panax ginseng (Araliaceae) & Fruits, root & $\begin{array}{l}\text { Saponins (ginsenosides, } \\
\text { panaxdiol, panaxtriole } \\
\text { and oleanolic acid) }\end{array}$ & $\begin{array}{c}\text { Adaptogenic } \\
\text { properties, } \\
\text { anti-arrhythmic. }\end{array}$ & $\begin{array}{l}\text { (Panax Ginseng. } \\
\text { Monograph, } \\
\text { 2009) }\end{array}$ \\
\hline 65 & Piper longum (Piperaceae) & Fruits & Alkaloids & Antioxidant. & $\begin{array}{c}\text { (Sunila and } \\
\text { Kuttan, 2004) }\end{array}$ \\
\hline 66 & $\begin{array}{c}\text { Rhodiola imbricate } \\
\text { (Crassulaceae) }\end{array}$ & Rhizomes & Phenolics & $\begin{array}{c}\text { Immunostimulating } \\
\text { properties. }\end{array}$ & $\begin{array}{l}\text { (Mishra et al, } \\
2008)^{88}\end{array}$ \\
\hline 67 & $\begin{array}{l}\text { Randia dumetorum } \\
\text { (Rubiaceae) }\end{array}$ & Fruits & Saponins, triterpenes & $\begin{array}{c}\text { Chlorosis, } \\
\text { anti-arthritic. }\end{array}$ & $\begin{array}{l}\text { (Satpute et al, } \\
2009)^{89}\end{array}$ \\
\hline 68 & $\begin{array}{c}\text { Silybum marianum } \\
\text { (Asteraceae) }\end{array}$ & Flowers & Flavonoid & Anti-oxidant. & $\begin{array}{l}\text { (Meeran et al, } \\
2006)^{90}\end{array}$ \\
\hline 69 & $\begin{array}{l}\text { Salicornia herbacea } \\
\text { (Chenopodiaceae) }\end{array}$ & Herb & Polysaccharides & Immunomodulator. & $(\operatorname{Im} \text { et al, 2006) })^{91}$ \\
\hline 70 & $\begin{array}{l}\text { Terminalia arjuna } \\
\text { (Combretaceae) }\end{array}$ & Leaves, bark & $\begin{array}{c}\text { Flavonoids, oligomeric } \\
\text { proanthocyanidins, } \\
\text { tannins } \\
\end{array}$ & $\begin{array}{c}\text { Cardiotonic, } \\
\text { diuretic, prescribed } \\
\text { for hypertension. }\end{array}$ & $\begin{array}{c}\text { (Halder et al, } \\
2009)^{92}\end{array}$ \\
\hline 71 & $\begin{array}{c}\text { Thuja occidentalis } \\
\text { (Arborvitae) }\end{array}$ & Leaves & Polysaccharides & Immunomodulator. & $\begin{array}{c}\text { (Gohla et al, } \\
1992)^{93}\end{array}$ \\
\hline 72 & $\begin{array}{c}\text { Tinospora cordifolia Miers. } \\
\text { (Menispermiaceae) }\end{array}$ & Entire herb & Alkaloidal constituents & $\begin{array}{c}\text { Hypoglycaemic } \\
\text { agent, anti-pyretic. }\end{array}$ & $(\text { Kirti et al, 2004) })^{94}$ \\
\hline 73 & Urena lobate (Malvaceae) & $\begin{array}{c}\text { Roots, } \\
\text { flowers } \\
\end{array}$ & Flavanoids & $\begin{array}{l}\text { Diuretic, emollient, } \\
\text { anti-spasmodic. }\end{array}$ & $\begin{array}{l}\text { (Rinku et al, } \\
2009)^{95}\end{array}$ \\
\hline 74 & $\begin{array}{l}\text { Viscum album } \\
\text { (Loranthaceae) }\end{array}$ & $\begin{array}{l}\text { Leaves and } \\
\text { young twigs, } \\
\text { berries }\end{array}$ & $\begin{array}{l}\text { Viscotoxins, } \\
\text { polyphenols, } \\
\text { polysaccharides }\end{array}$ & Anti-tumor effect. & $\begin{array}{l}\text { (Elluru et al, } \\
2007)^{96}\end{array}$ \\
\hline
\end{tabular}


Farid A. Badria. / International Journal of Research in Pharmaceutical and Nano Sciences. 9(2), 2020, 46-71.

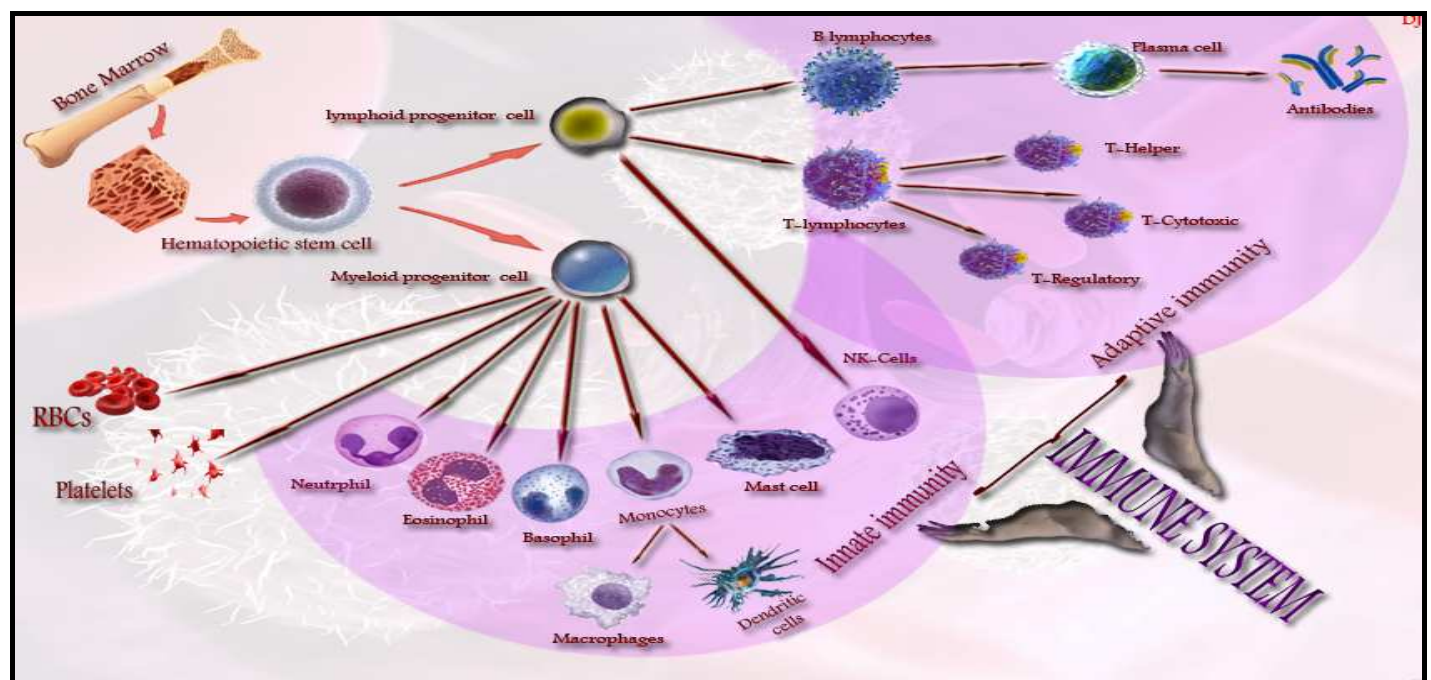

Figure No.1: Origin and types of immune system cells (Created by Our own Team)

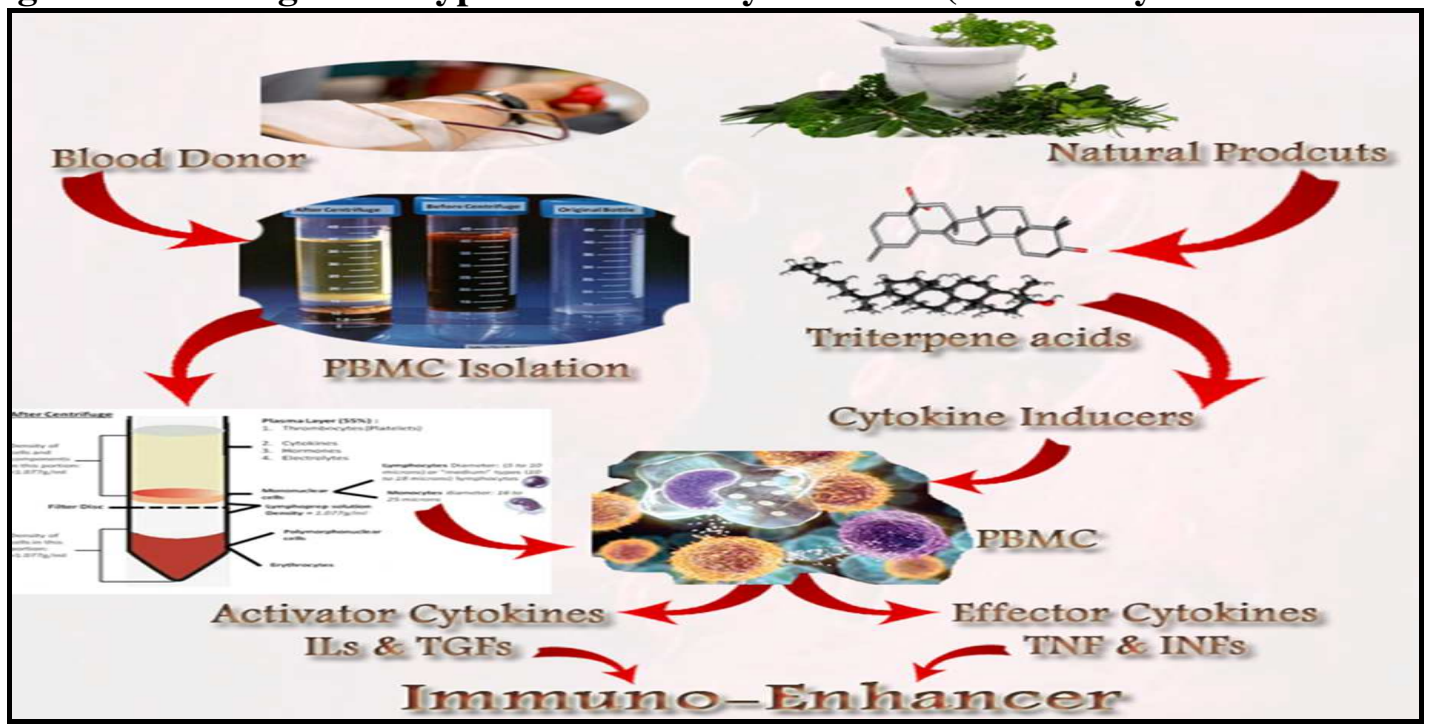

Figure No.2: General aim of the study (Created by Our own Team)

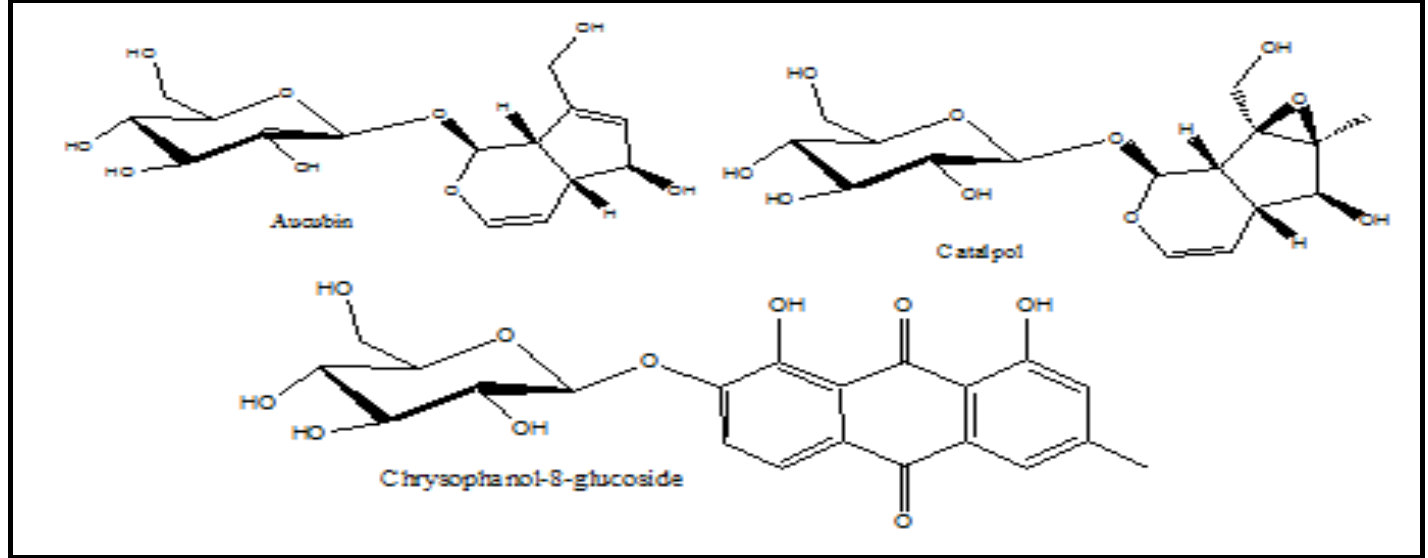

Figure No.3: Structure of aucubin and catalpol, two of the most common iridoids in the plant kingdom and chrysophanol-8-glucoside an immunomodulators anthraquinone glycosides

Available online: www.uptodateresearchpublication.com March - April 
Farid A. Badria. / International Journal of Research in Pharmaceutical and Nano Sciences. 9(2), 2020, 46-71.<smiles>[R]OC1C(=O)c2c(O)c(OC)c(OC)c(O)c2OC1c1ccc(O)cc1</smiles>

Figure No.4: Structure of eupalitin and centaureidin, flavonoids showing immunomodulatory activity

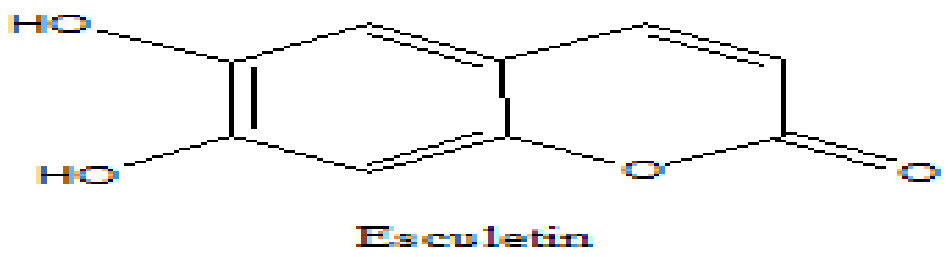

Figure No.5: Structure of immunomodulatory coumarin (Esculetin)<smiles>O=C(/C=C/C=C/c1ccc2c(c1)OCO2)N1CCCCC1</smiles>

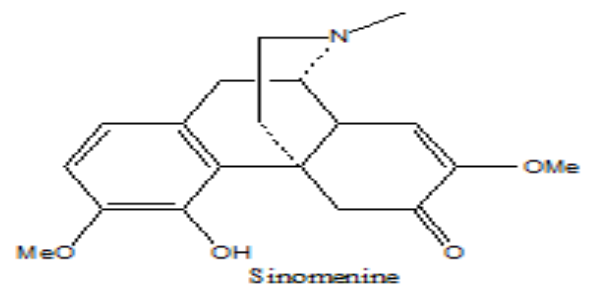

Figure No.6: Structure of some plant alkaloids with immunomodulatory activity Thiosulfinates<smiles>[R]SS([R])=O</smiles>

Thiosulfinates<smiles>C=CCS(=O)CC=C</smiles>

Allicin

Figure No.7: Structure of Allicinthiosulfinate derived from garlic which has immunomodulatory activity

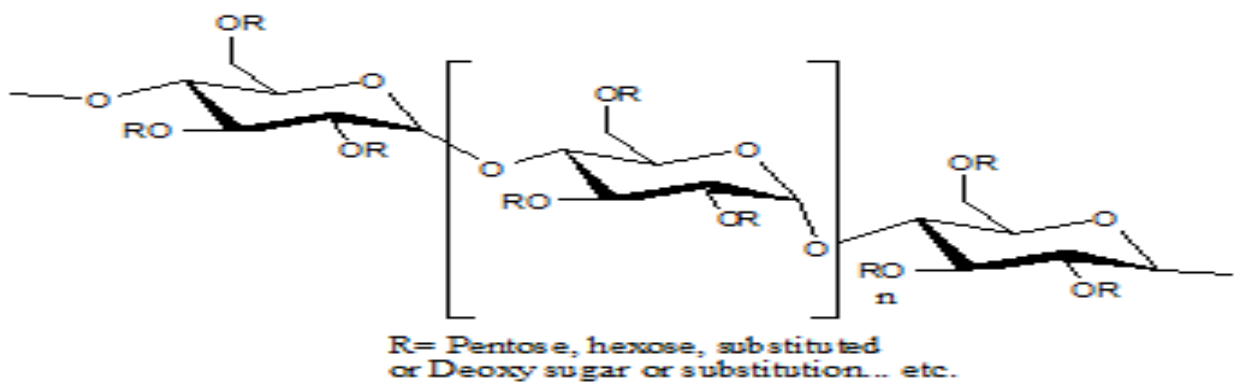

Figure No.8: Structure of main building unit of polysaccharides

Available online: www.uptodateresearchpublication.com March - April 
Farid A. Badria. / International Journal of Research in Pharmaceutical and Nano Sciences. 9(2), 2020, 46-71.
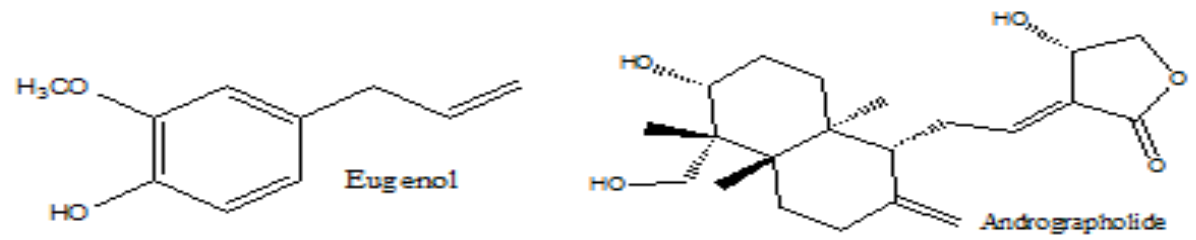

Figure No.9: Structure of eugenol and andrographolide which have immunomodulatory activities
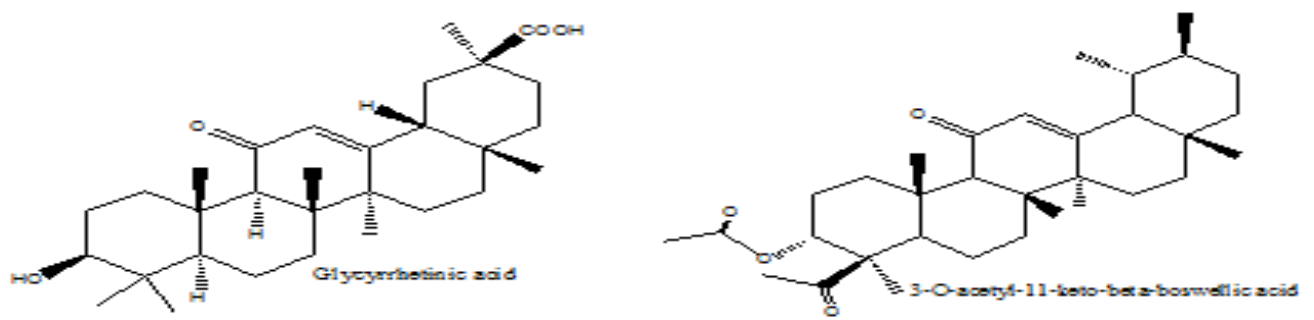

Figure No.10: Structure of glycyrrhetinic acid and boswellic acid "AKBA", triterpene acids isolated from Glycyrrhiza glabra and Boswellia spp

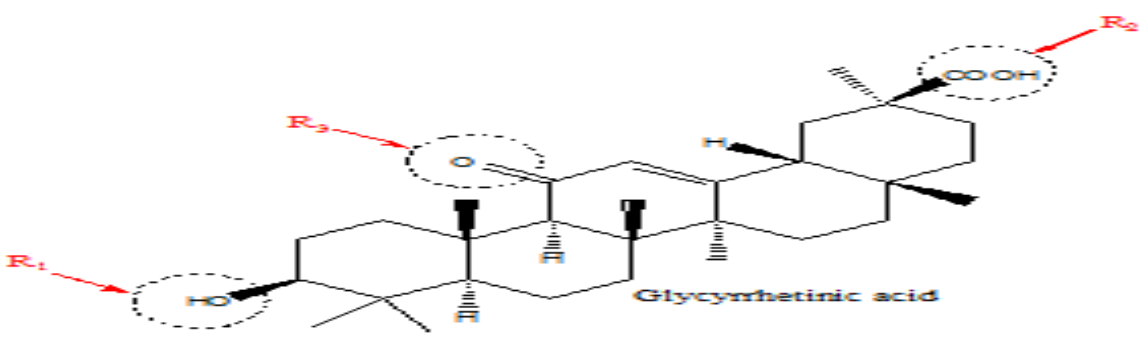

Figure No.11: Main sites of modification on glycyrrhetinic acid
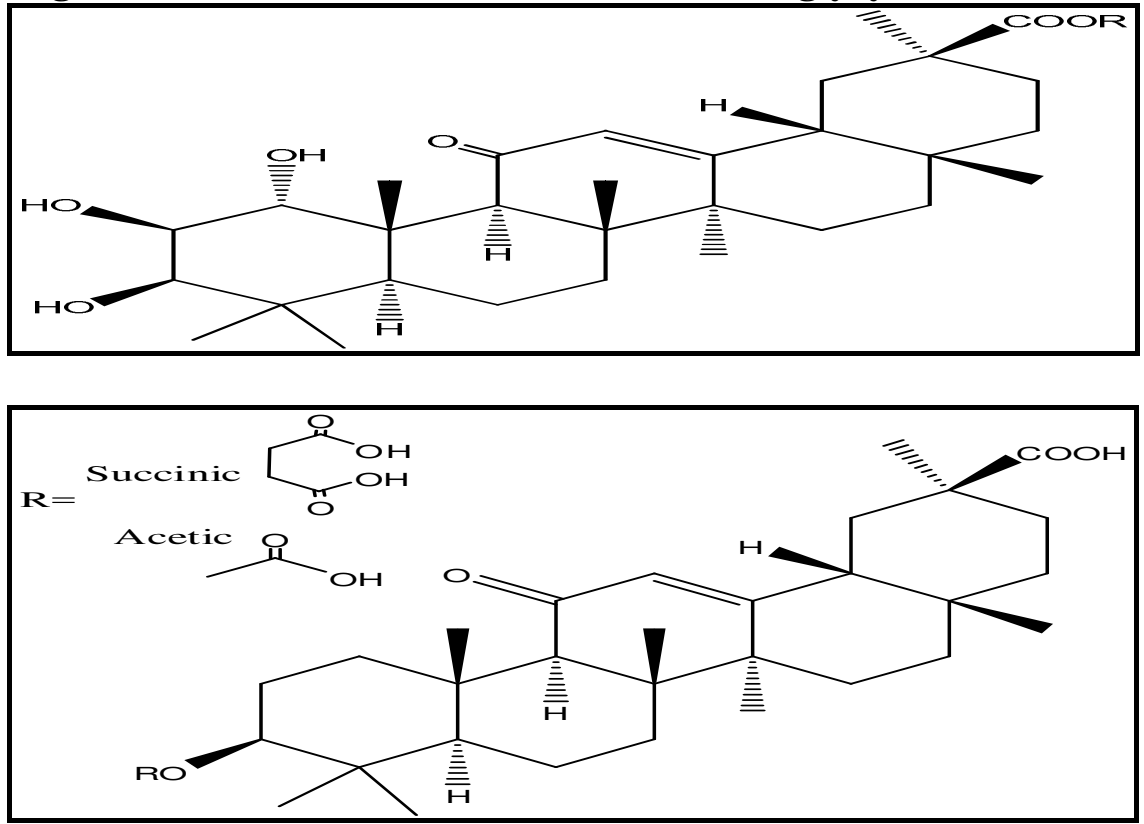

Available online: www.uptodateresearchpublication.com March - April 
Farid A. Badria. / International Journal of Research in Pharmaceutical and Nano Sciences. 9(2), 2020, 46-71.
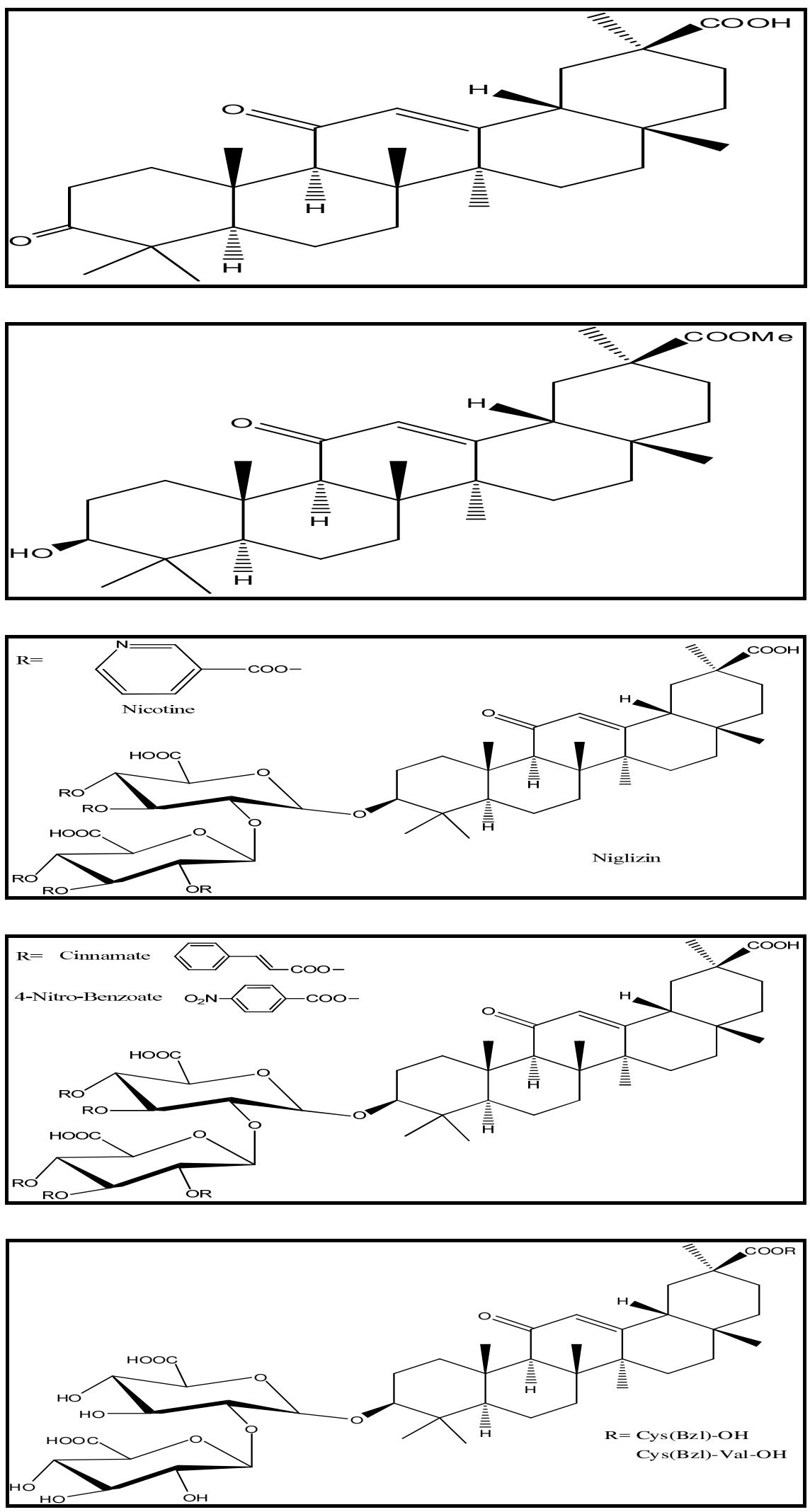

Available online: www.uptodateresearchpublication.com March - April 
Farid A. Badria. / International Journal of Research in Pharmaceutical and Nano Sciences. 9(2), 2020, 46-71.
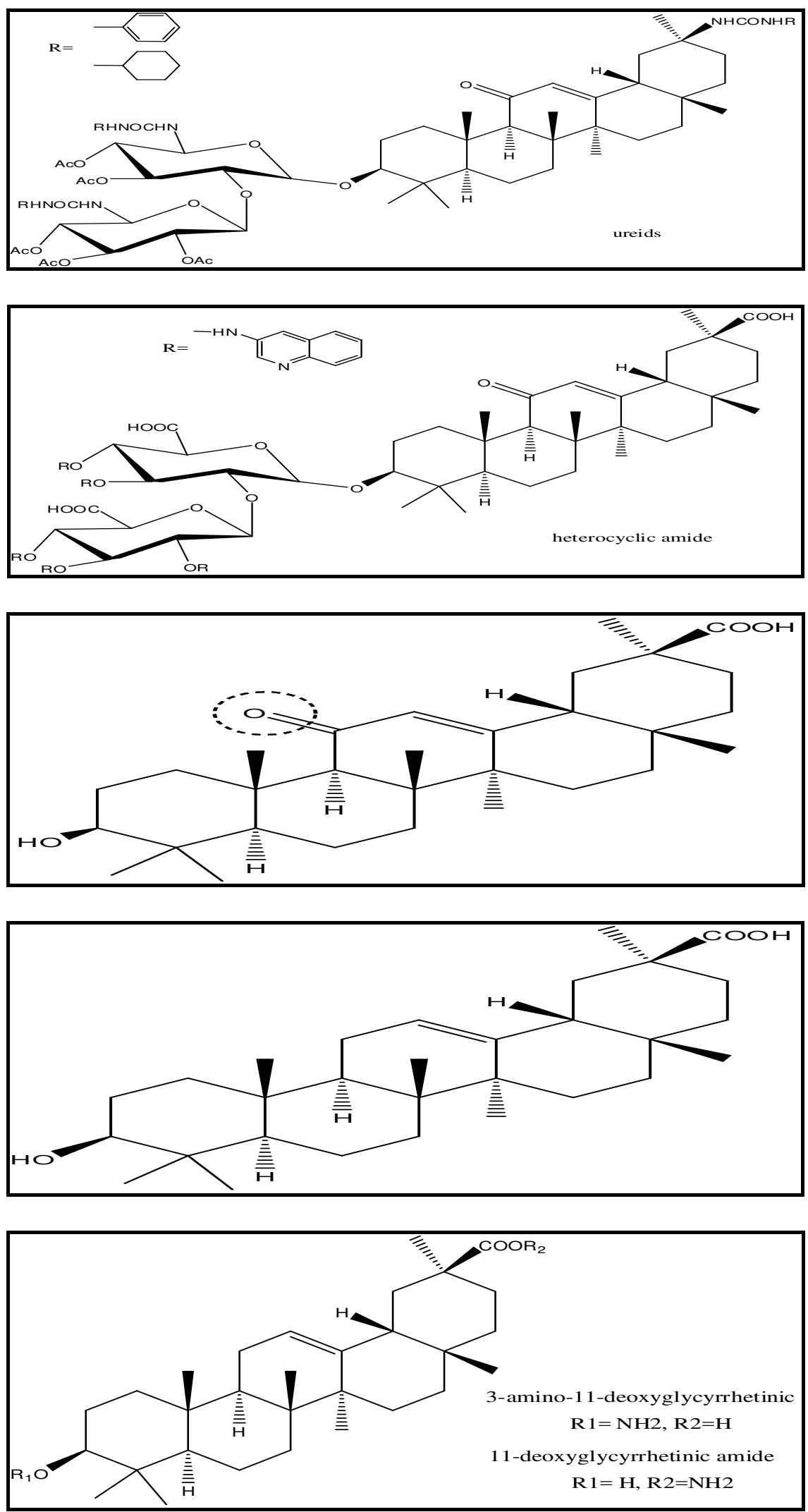

Available online: www.uptodateresearchpublication.com March - April 
Farid A. Badria. / International Journal of Research in Pharmaceutical and Nano Sciences. 9(2), 2020, 46-71.

\section{CONCLUSION}

Immunomodulation either using natural products or their semisynthetic derivatives can provide support to achieve the healthy state in normal or diseased patients. Also, agents that can affect immunity in a situation of immune impairment can then be used as co-therapy with chemotherapy (Wagner et al, $2012)^{113}$. Bone marrow is a sensitive target especially to cytotoxic agents due to high degree of cell proliferation. In fact, under immunosuppression therapy, bone marrow is the most affected organ which in turn results in leucopenia and thrombocytopenia (Bafna and Mishra, 2006) ${ }^{114}$. Triterpenoidal and steroidal saponins (Yesilada et al, 2005) $)^{115}$ are proven to have versatile pharmacological activities, such as being antihepatotoxic, molluscicidal, antifungal, cytotoxic, antiviral and immunomodulator.

Immunomodulation activities of terpenoids such as glycyrrhizin, ursolic acid and oleanolic acid, have been reported (Raphael and Kuttan, 2003) ${ }^{116}$. Many medicinal plants can exert immunomodulatory activity in experimental models at a specific dose. Different types of in vivo and in vitro screening models both have been employed to study that activity. Some medicinal plant extracts can produce immunostimulation (e.g., Glycyrrhiza glabra, Ocimum sanctum), and others can produce immunosuppression (Alternanthera tenella). Finally, various phytochemical active isolates (e.g., glycosides, terpenoids, saponins, steroids, alkaloids, coumarins, and flavonoids) may exhibit a wide range of immunomodulatory activities.

Immunity is the body ability to identify and resist harmful pathogen. The immune system has a multilayered construction, with several stages of defenses. The skin is the first barrier of immunity. In recent years, there is an upsurge in the clinical use of natural products or their derivatives due to being free from serious toxic effects and better efficacy. Additionally, steady increase in the resistant microorganism strains to antibiotics and serious averse manifestations brought about by the synthetic drugs has incited researchers to search for

Available online: www.uptodateresearchpublication.com natural immunomodulators to fight different infections (Shukla et al, 2014) ${ }^{117}$.

Mechanisms of action of an immunomodulator goes mainly through stimulation of phagocytosis, activation of macrophages, stimulation of lymphoid cells, enhancement of nonspecific cellular immune function, increasing natural killer cell numbers and nonspecific mediators of immune system, increasing the production of antigenspecific antibodies, counter the effect on leukopenia induced by chemotherapy, and increasing total counts of circulating white blood cells and induction of variety of cytokine levels.

\section{ACKNOWLEDGEMENT}

I would like to thank my student Mr. Diaaeldin M. Elimam, and my colleagues, Drs. Hosam M. Zaghloul, Fatma M. Abdel Bar, for their valuable contribution and support during the practical part of this study. Also, I would like to thank FAB-Lab, Liver Research Lab, and those who maintain it.

\section{CONFLICT OF INTEREST}

We declare that we have no conflict of interest.

\section{REFERENCES}

1. Badria F A, Abu-Karam M, Mikhaeil B R, Maatooq G T and Amer M M. Anti-herpes activity of isolated compounds from frankincense, Biosciences Biotechnology Research Asia, 1(1), 2003, 1-10.

2. Mikhaeil B R, Maatooq G T, Badria F A, Amer $M \quad M$. Chemistry and immunomodulatory activity of frankincense oil, Z. Naturforsch. C, 58(3-4), 2003, 230238.

3. Badria F A, Mikhaeil B R, Maatooq G T, Amer M M. Immunomodulatory triterpenoids from the oleogum resin of Boswellia carterii Birdwood, Zeitschrift Fur Naturforsch. C, 58(7-8), 2003, 505-516.

4. Badria F A. New type of tyrosinase inhibitors from natural products as potential treatments for hyperpigmentation, Bollettino Chimico Farmaceutico, 140(4), 2001, 267-271.

March - April

61 
Farid A. Badria. / International Journal of Research in Pharmaceutical and Nano Sciences. 9(2), 2020, 46-71.

5. Badria F A. Is man helpless against cancer? An environmental approach: antimutagenic agents from Egyptian food and medicinal preparations, Cancer letters, 84(1), 1994, 1-5.

6. Ibrahim A, Sobeh M, Ismail A, Alaa A, Sheashaa H, Sobh M, Badria F. Free-B-Ring flavonoids as potential lead compounds for colon cancer therapy, Molecular and Clinical Oncology, 2(4), 2014, 581-585.

7. Badria F, Mabed M, El-Awadi M, Abou-Zeid L, Al-Nashar E and Hawas S. Immune modulatory potentials of antineoplaston A-10 in breast cancer patients, Cancer Letters, 157(1), 2000a, 57-63.

8. Badria F, Mabed M, Khafagy W and AbouZeid L. Potential utility of antineoplaston A10 levels in breast cancer, Cancer Letters, 155(1), 2000b, 67-70.

9. Elimam D M A, Uddin Ibrahim A S, Liou G I and Badria F A. Olive and ginkgo extracts as potential cataract therapy with differential inhibitory activity on aldose reductase, Drug Discoveries and Therapeutics, 11(1), 2017, 41-46.

10. Badria F, Abou-Mohamed G, El-Mowafy A, Masoud A and Salama O. Mirazid: A new schistosomicidal drug, Pharmaceutical Biology, 39(2), 2001, 127-131.

11. El-Attar E,Galala A, Gohar A and Badria F. Cycloartane Glycoside: A New Lactate Dehydrogenase Inhibitor, from the Aerial part of Agriculture Waste of Watermelon, World Journal of Pharmaceutical Sciences, 3(4), 2015, 689-695.

12. Abdel Bar F M, Khanfar M A, Elnagar A Y, Liu H, Zaghloul A M, Badria F A, Sylvester P W, Ahmad K F, Raisch K P and El Sayed K A. Rational design and semisynthesis of betulinic acid analogues as potent topoisomerase inhibitors, Journal of Natural Products, 72(9), 2009, 1643-1650.

13. El-Naggar M H, Mira A, Bar F M A, Shimizu K, Amer M M and Badria F A. Synthesis, docking, cytotoxicity, and LTA4H inhibitory activity of new gingerol derivatives as

Available online: www.uptodateresearchpublication.com potential colorectal cancer therapy, Bioorganic and Medicinal Chemistry, 25(3), 2017, 1277-1285.

14. Abdel Bar F M, Khanfar M A, Elnagar A Y, Badria F A, Zaghloul A M, Ahmad K F, Sylvester P W and El K S. Design and pharmacophore modeling of biaryl methyl eugenol analogs as breast cancer invasion inhibitors, Bioorganic and Medicinal Chemistry, 18(2), 2010, 496-507.

15. El-Senduny F F, Badria F A, El-Waseef A M, Chauhan S C and Halaweish F. Approach for chemosensitization of cisplatin-resistant ovarian cancer by cucurbitacin B, Tumor Biology, 37(1), 2016, 685-698.

16. Abdel Bar F M, Elimam D M, Mira A S, ElSenduny F F and Badria F A. Derivatization, molecular docking and in vitro acetylcholinesterase inhibitory activity of glycyrrhizin as a selective anti-Alzheimer agent, Natural Product Research, 33(18), 2019, 2591-2599.

17. Haskell-Ramsay C F, et al. The Acute Effects of Caffeinated Black Coffee on Cognition and Mood in Healthy Young and Older Adults, Nutrition, 10(10), 2018, e1386.

18. Badria F A and Ahmed M H. Multi-targets drugs: A new therapeutic approach, International Journal of Research in Pharmaceutical and Nano Sciences, 7(5), 2018, 205-213.

19. Hofmeyr S A. An interpretative introduction to the immune system, Des. Princ. Immune Syst. Other Distrib. Auton. Syst, 3, 2001, 2836.

20. Organ J M. Lippincott Williams and Wilkins Atlas of Anatomy, JAMA J. Am. Med. Assoc, 301(17), 2009, 1737-1844.

21. Janeway C A, Travers P, Walport M, Capra J D. Immunobiology: the immune system in health and disease, $5^{\text {th }}$ Edition, 2005, 1-884.

22. Wilasrusmee C, Kittur S, Shah G, Siddiqui J, Bruch D, Wilasrusmee S, Kittur D S. Immunostimulatory effect of Silybum 
Farid A. Badria. / International Journal of Research in Pharmaceutical and Nano Sciences. 9(2), 2020, 46-71.

Marianum (milk thistle) extract, Med. Sci. Monit, 8(11), 2002, BR439-BR443.

23. Liou C J, Li M L, Tseng J. Intraperitoneal injection of ginseng extract enhances both immunoglobulin and cytokine production in mice, Am. J. Chin. Med, 32(1), 2004, 75-88.

24. Melchart D, Linde K, Worku F, Bauer R, Wagner H. Immunomodulation with Echinacea-a systematic review of controlled clinical trials, Phytomedicine, 1(3), 1994, 245-254.

25. Billiau A, Matthys P. Modes of action of Freund's adjuvants in experimental models of autoimmune diseases, J. Leukoc. Biol, 70(6), 2001, 849-860.

26. Killestein J, Hoogervorst E L J, Reif M, Blauw B, Smits M, Uitdehaag B M J, Nagelkerken L, Polman C $\mathrm{H}$. Immunomodulatory effects of orally administered cannabinoids in multiple sclerosis, J. Neuroimmunol, 137(1-2), 2003, 140-143.

27. Ordway D, Hohmann J, Viveiros M, Viveiros A, Molnar J, Leandro C, Arroz M J, Gracio M A, Amaral L. Carpobrotus edulis methanol extract inhibits the MDR efflux pumps, enhances killing of phagocytosed S. aureus and promotes immune modulation, Phytother. Res, 17(5), 2003, 512-519.

28. Sharififar F, Pournourmohammadi S, Arabnejad M. Immunomodulatory activity of aqueous extract of Achillea wilhelmsii C. Koch in mice, Indian J. Exp. Biol, 47(8), 2009, 668-671.

29. Hamman J H. Composition and applications of Aloe vera leaf gel, Molecules, 13(8), 2008, 1599-1616.

30. Varma A, Padh H, Shrivastava N. Andrographolide: A new plant-derived antineoplastic entity on horizon, Evid. Based. Complement. Alternat. Med, 2011, Article ID 815390, 2011, 9.

31. Dashputre N L, Naikwade N S. Immunomodulatory activity of Abutilon

Available online: www.uptodateresearchpublication.com indicum linn on albino mice, Int. J. Pharm. Sci. Res, 1(3), 2010, 178-184.

32. Bopana N, Saxena S. Asparagus racemosusethnopharmacological evaluation and conservation needs, J. Ethnopharmacol, 110(1), 2007, 1-15.

33. Guerra R N M, Pereira H A W, Silveira L M S, Olea R S G. Immunomodulatory properties of Alternanthera tenella Colla aqueous extracts in mice, Braz. J. Med. Biol. Res, 36(9), 2003, 1215-1219.

34. Lu Y, Fan J, Zhao Y, Chen S, Zheng X, Yin $\mathrm{Y}, \mathrm{Fu} \mathrm{C}$. Immunomodulatory activity of aqueous extract of Actinidia macrosperma, Asia Pac. J. Clin. Nutr, 16(1), 2007, 261-265.

35. Ismail S, Asad M. Immunomodulatory activity of Acacia catechu, Indian J. Physiol. Pharmacol, 53(1), 2009, 25-33.

36. Jeong S C, Jeong Y T, Yang B K, Song C H. Chemical characteristics and immunostimulating properties of biopolymers extracted from Acanthopanax sessiliflorus, $J$. Biochem. Mol. Biol, 39(1), 2006, 84-90.

37. Hung L C, Lin C C, Hung S K, Wu B C, Jan $\mathrm{M} \mathrm{D}$, Liou S H, Fu S L. A synthetic analog of alpha-galactosylceramide induces macrophage activation via the TLR4signaling pathways, Biochem. Pharmacol, 73(12), 2007, 1957-1970.

38. Zheng W, Chen C, Cheng Q, Wang Y, Chu C. Oral administration of exopolysaccharide from Aphanothece halophytica (Chroococcales) significantly inhibits influenza virus (H1N1)-induced pneumonia in mice, Int. Immunopharmacol, 6(7), 2006, 1093-1099.

39. Cherng J M, Chiang W, Chiang L C. Immunomodulatory activities of common vegetables and spices of Umbelliferae and its related coumarins and flavonoids, Food Chem, 106(3), 2008, 944-950.

40. Kobayashi H, De Mejia E. The genus Ardisia: a novel source of health-promoting compounds and phytopharmaceuticals, $J$. Ethnopharmacol, 96(3), 2005, 347-354.

March - April

63 
Farid A. Badria. / International Journal of Research in Pharmaceutical and Nano Sciences. 9(2), 2020, 46-71.

41. Wang C, Tao W, Wang Y, Bikow J, Lu B, Keating A, Verma S, Parker T G, Han R, Wen $\mathrm{X}$ Y. Rosuvastatin, identified from a zebrafish chemical genetic screen for antiangiogenic compounds, suppresses the growth of prostate cancer, Eur. Urol, 58(3), 2010, 418-426.

42. Noori S, Naderi G A, Hassan Z M, Habibi Z, Bathaie $S$ Z, Hashemi S M M. Immunosuppressive activity of a molecule isolated from Artemisia annua on DTH responses compared with cyclosporin A, Int. Immunopharmacol, 4(10-11), 2004, 13011306.

43. Steinbach W J, Stevens D A. Review of newer antifungal and immunomodulatory strategies for invasive aspergillosis, Clin. Infect. Dis, 37(3), 2003, S157-S187.

44. Ghaisas M M, Shaikh S A, Deshpande A D. Evaluation of the immunomodulatory activity of ethanolic extract of the stem bark of Bauhinia variegata Linn, Int. J. Green Pharm, 3(1), 2009, 70-74.

45. Ballarin L. Immunobiology of compound ascidians, with particular reference to Botryllus schlosseri: state of art, Inv. Surv. J, 5(1), 2008, 54-74.

46. Chang S L, Chiang Y M, Chang C L T, Yeh H H, Shyur L F, Kuo Y H, Wu T K, Yang W C. Flavonoids, centaurein and centaureidin, from Bidens pilosa, stimulate IFN-gamma expression, J. Ethnopharmacol, 112(2), 2007, 232-236.

47. Mungantiwar A A, Nair A M, Shinde U A, Dikshit V J, Saraf M N, Thakur V S, Sainis K B. Studies on the immunomodulatory effects of Boerhaavia diffusa alkaloidal fraction, $J$. Ethnopharmacol, 65(2), 1999, 125-131.

48. Sredni B, Kalechman Y, Albeck M, Gross O, Aurbach D, Sharon P, Sehgal S N, Gurwith M J, Michlin H. Cytokine secretion effected by synergism of the immunomodulator AS101 and the protein kinase $\mathrm{C}$ inducer bryostatin, Immunology, 70(4), 1990, 473-477.

49. Johnston M F, Hays R D, Hui K K. Evidencebased effect size estimation: an illustration using the case of acupuncture for cancerrelated fatigue. BMC Complement, Altern. Med, 9(1), 2009, 1.

50. Bafna A, Mishra S. Antioxidant and immunomodulatory activity of the alkaloidal fraction of Cissampelos pareira linn, Sci. Pharm, 78(1), 2010, 21-31.

51. Gaur K, Kori M L, Nema R K. Comparative screening of immunomodulatory activity of hydro-alcoholic extract of Hibiscus rosasinensis Linn. and ethanolic extract of Cleome gynandra Linn, Glob. J. Pharmacol, 3(2), 2009, 85-89.

52. Thakur M, Bhargava S, Dixit V K. Immunomodulatory activity of Chlorophytum borivilianum Sant. F, Evidence-Based Complement. Altern. Med, 4(4), 2007, 419423.

53. Tanaka T, Sugiura H, Inaba R, Nishikawa A, Murakami A, Koshimizu K, Ohigashi H. Immunomodulatory action of citrus auraptene on macrophage functions and cytokine production of lymphocytes in female BALB/c mice, Carcinogenesis, 20(8), 1999, 14711476.

54. Mani S, Lawson J W. In vitro modulation of inflammatory cytokine and IgG levels by extracts of Perna canaliculus, $B M C$ Complement. Altern. Med, 6(1), 2006, 1.

55. Bhatt P R, Pandya K B, Sheth N R. Camellia sinensis (L): the medicinal beverage: a review, Int. J. Pharm. Sci. Rev. Res, 3(2), 2010, 3-6.

56. Mali R G, Hatapakki B C. An in vitro study of effect of Centella asiatica on phagocytosis by human neutrophils, Int. J. Pharm. Sci. Nanotechnol, 1(3), 2008, 297-302.

57. Costa J F O, David J P L, David J M, Giulietti A M, Queiroz L P, Santos R R, Soares M B P. Immunomodulatory activity of extracts from Cordia superba Cham. and Cordia rufescens A. DC.(Boraginaceae), plant species native from Brazilian Semi-arid, Rev. Bras. Farmacogn, 18(1), 2008, 11-15.

March - April

64 
Farid A. Badria. / International Journal of Research in Pharmaceutical and Nano Sciences. 9(2), 2020, 46-71.

58. Ebringerova A, Hromadkova Z, Hribalova V, Hirsch J. An Immunomodulating Pectic Arabinogalactan from Roots, Chem. Pap, 56(5), 2002, 320-325.

59. Sugumaran M, Robinson W E. Bioactive dehydrotyrosyl and dehydrodopyl compounds of marine origin, Mar. Drugs, 8(12), 2010, 2906-2935.

60. Hsu C H, Sun H L, Sheu J N, Ku M S, Hu C $M$, Chan Y, Lue K H. Effects of the immunomodulatory agent Cordyceps militaris on airway inflammation in a mouse asthma model, Pediatr. Neonatol, 49(5), 2008, 171178.

61. Pradhan D, Panda P K, Tripathy G. Evaluation of Immunomodulatory activity of the methanolic extract of Couroupita guianensis Aubl flowers in rats, Nat. Prod. Radiance, 8(1), 2009, 37-42.

62. Zvetkova E, Wirleitner B, Tram N T, Schennach H, Fuchs D. Aqueous extracts of Crinum latifolium (L.) and Camellia sinensis show immunomodulatory properties in human peripheral blood mononuclear cells, Int. Immunopharmacol, 1(12), 2001, 2143-2150.

63. Amirghofran Z, Azadbakht M, Karimi M H. Evaluation of the immunomodulatory effects of five herbal plants, J. Ethnopharmacol, 72(1-2), 2000, 167-172.

64. Senchina D S, McCann D A, Asp J M, Johnson J A, Cunnick J E, Kaiser M S, Kohut $M$ L. Changes in immunomodulatory properties of Echinacea spp. root infusions and tinctures stored at $4 \mathrm{C}$ for four days, Clin. Chim. Acta, 355(1-2), 2005, 67-82.

65. Jayathirtha M G, Mishra S H. Preliminary immunomodulatory activities of methanol extracts of Eclipta alba and Centella asiatica, Phytomedicine, 11(4), 2004, 361-365.

66. Sandeep B. Patel, Nilofar S. Naikwade, Chandrakant S. Magdum. Review on Phytochemistry and Pharmacological Aspects of Euphorbia hirta Linn, Asian J. Pharm. Res. Heal. Care, 1(1), 2009, 113-133 .

Available online: www.uptodateresearchpublication.com
67. Ganju L, Karan D, Chanda S, Srivastava K K, Sawhney R C, Selvamurthy W. Immunomodulatory effects of agents of plant origin, Biomed. Pharmacother, 57(7), 2003, 296-300.

68. Habijanic J, Berovic M, Wraber B, Hodzar D, Boh B. Immunostimulatory effects of fungal polysaccharides from Ganoderma lucidum submerged biomass cultivation, Food Technol. Biotechnol, 39(4), 2001, 327-332.

69. Malik J K, Manvi F V, Nanjwade B K, Alagawadi K R, Singh S. Immunomodulatory activity of Gymnema sylvestre R. Br. leaves on in-vitro human neutrophils, J. Pharm. Res, 2, 2009, 1284-1286.

70. Amirghofran Z, Azadmehr A, Javidnia K. Haussknechtia elymatica: a plant with immunomodulatory effects, Iran. J. Immunol, 4(1), 2007, 26-31.

71. Sharififar F, Pournourmohammadi S, Rastegarianzadeh R, Ranjbaran O, Purhemmaty A. Immunomodulatory activity of aqueous extract of Heracleum persicum Desf. in mice, Iran. J. Pharm. Res, 8(4), 2010, 287-292.

72. Jain S, Bhagwat D, Jat R C, Bhardwaj S. The immunomodulation potential of Hyptis suaveolens, IJPRD, 1(11), 2005, 1-6.

73. Caifa Chen W Z, Gao X, Xiang X. Aqueous Extract of Inonotus obliquus (Fr.) Pilat (Hymenochaetaceae) Significantly Inhibits the Growth of Sarcoma 180 by Inducing Apoptosis, Am J Pharmacol Toxicol, 2(1), 2007, 10-17.

74. Davicino R, Mattar A, Casali Y, Porporatto C, Correa $\mathrm{S} \mathrm{G}$, Micalizzi $\mathrm{B}$. In vivo immunomodulatory effects of aqueous extracts of Larrea divaricata Cav, Immunopharmacol. Immunotoxicol, 29(3-4), 2007, 351-366.

75. Gan L, Zhang S H, Liu Q, Xu H B. A polysaccharide-protein complex from Lycium barbarum upregulates cytokine expression in human peripheral blood mononuclear cells, Eur. J. Pharmacol, 471(3), 2003, 217-222.

March - April 65 
Farid A. Badria. / International Journal of Research in Pharmaceutical and Nano Sciences. 9(2), 2020, 46-71.

76. Deshpande J R, Choudhari A A, Mishra M R, Meghre V S, Wadodkar S G, Dorle, A K. Beneficial effects of Lagenaria siceraria (Mol.) Standley fruit epicarp in animal models, Indian J. Exp. Biol, 46(4), 2008, 234242.

77. De Souza Reis L S, Frazatti-Gallina N M, De Lima Paoli R, Giuffrida R, Albas A, Oba E, Pardo P E. Efficiency of Matricaria chamomilla $\mathrm{CH} 12$ and number of doses of rabies vaccine on the humoral immune response in cattle, J. Vet. Sci, 9(4), 2008, 433435.

78. Ferreira A P, Soares G L G, Salgado C A, Goncalves L S, Teixeira F M, Teixeira H C, Kaplan M A C. Immunomodulatory activity of Mollugo verticillata L, Phytomedicine, 10(2-3), 2003, 154-158.

79. Bharani S E R, Asad M, Dhamanigi S S, Chandrakala G K. Immunomodulatory activity of methanolic extract of Morus alba Linn. (mulberry) leaves, Pak. J. Pharm. Sci, 23(1), 2010, 63-68.

80. Gupta A, Gautam M K, Singh R K, Kumar M V, Rao C V, Goel R K, Anupurba S. Immunomodulatory effect of Moringa oleifera Lam, extract on cyclophosphamide induced toxicity in mice, Indian J Exp Biol, 48(11), 2010, 1157-1160.

81. Shah A S, Wakade A S, Juvekar A R. Immunomodulatory activity of methanolic extract of Murraya koenigii (L) Spreng. Leaves, Indian J. Exp. Biol, 46(7), 2008, 505509.

82. Kannan M, Singh A, Kumar $T$ T A, Jegatheswari P, Subburayalu S. Studies on immuno-bioactivities of Nyctanthes arbortristis (Oleaceae), African J. Microbiol. Res, 1(6), 2007, 88-91.

83. Vaghasiya J, Datani M, Nandkumar K, Malaviya S, Jivani N. Comparative evaluation of alcoholic and aqueous extracts of Ocimum sanctum for immunomodulatory activity, Int $J$ Pharm Biol Res, 1(1), 2010, 25-29.

Available online: www.uptodateresearchpublication.com
84. Kumar D S S, Lau C S, Wan J M F, Yang D, Hyde K D. Immunomodulatory compounds from Pestalotiopsis leucothes, an endophytic fungus from Tripterygium wilfordii, Life Sci, 78(2), 2005, 147-156.

85. Smit H F. Picrorhiza scrophulariiflora, from traditional use to immunomodulatory activity, 2001, 1-174.

86. Panax Ginseng. Monograph, Altern. Med. Rev, 14(2), 2009, 172-176.

87. Sunila E S, Kuttan G. Immunomodulatory and antitumor activity of Piper longum Linn. and piperine, J. Ethnopharmacol, 90(2-3), 2004, 339-346.

88. Mishra K P, Padwad Y S, Dutta A, Ganju L, Sairam M, Banerjee P K, Sawhney R C. Aqueous extract of Rhodiola imbricata rhizome inhibits proliferation of an erythroleukemic cell line K-562 by inducing apoptosis and cell cycle arrest at G2/M phase, Immunobiology, 213(2), 2008, 125-131.

89. Satpute K L, Jadhav M M, Karodi R S, Katare Y S, Patil M J, Rub R, Bafna A R. Immunomodulatory activity of fruits of Randia dumetorum Lamk, J. Pharmacogn. Phyther, 1(3), 2009, 36-40.

90. Meeran S M, Katiyar S, Elmets C A, Katiyar S K. Silymarin inhibits UV radiation-induced immunosuppression through augmentation of interleukin-12 in mice, Mol. Cancer Ther, 5(7), 2006, 1660-1668.

91. Im S A, Kim K, Lee C K. Immunomodulatory activity of polysaccharides isolated from Salicornia herbacea, Int. Immunopharmacol, 6(9), 2006, 1451-1458.

92. Halder S, Bharal N, Mediratta P K, Kaur I, Sharma K K. Anti-inflammatory, immunomodulatory and antinociceptive activity of Terminalia arjuna Roxb bark powder in mice and rats, Indian J. Exp. Biol, 47(7), 2009, 577-583.

93. Gohla S H, Zeman R A, Bogel M, Jurkiewicz E, Schrum S, Haubeck H D, Schmitz H, Hunsmann G, Neth R D. Modification of the in vitro replication of the human

March - April

66 
Farid A. Badria. / International Journal of Research in Pharmaceutical and Nano Sciences. 9(2), 2020, 46-71.

immunodeficiency virus HIV-1 by TPSg, a polysaccharide fraction isolated from the Cupressaceae Thuja occidentalis L. (Arborvitae), In: Modern Trends in Human Leukemia IX, Springer, 35, 1992, 140-149.

94. Kirti S, Mishra N P, Singh J. Tinospora cardifolia, a reservoir plant for therapeutic applicant, Indian J. of. Tradit. Knowl, 3(3), 2004, 257-270.

95. Rinku M, Prasanth V V, Parthasarathy G. Immunomodulatory activity of the methanolic extract of Urena lobata Linn, Internet $J$. Pharmacol, 7(1), 2009, 1-4.

96. Elluru S, Duong Van Huyen J P, Wootla B, Delignat S, Prost F, Negi V S, Kaveri S V. Tumor regressive effects of viscum album preparations exploration of immunomodulatory mechanisms, Medicina (B. Aires), 67(2), 2007, 85-89.

97. Mehrotra S, Singh V K, Agarwal S S, Maurya R, Srimal R C. Antilymphoproliferative activity of ethanolic extract of Boerhaavia diffusa roots, Exp. Mol. Pathol, 72(3), 2002, 236-242.

98. Davis L, Kuttan G. Immunomodulatory activity of Withania somnifera, $J$. Ethnopharmacol, 71(1-2), 2000, 193-200.

99. Bhattacharya S K, Bhattacharya A, Chakrabarti A. Adaptogenic activity of Siotone, a polyherbal formulation of Ayurvedic rasayanas, Indian J. Exp. Biol, 38(2), 2000, 119-128.

100. Makare N, Bodhankar S, Rangari V. Immunomodulatory activity of alcoholic extract of Mangifera indica L. in mice, $J$. Ethnopharmacol, 78(2-3), 2001, 133-137.

101. Leung K, Leung $P$, Kong L, Leung $P$. Immunomodulatory effects of esculetin $(6,7-$ dihydroxycoumarin) on murine lymphocytes and peritoneal macrophages, Cell. Mol. Immunol, 2(3), 2005, 181-188.

102. Jafarian A, Ghannadi A, Elyasi A. The effects of Allium hirtifolium Boiss. on cellmediated immune response in mice, Iran. J. Pharm. Res, 2(1), 2010, 51-55.

Available online: www.uptodateresearchpublication.com
103. Alamgir M, Uddin S J. 8. Recent advances on the ethnomedicinal plants as immunomodulatory agents, Debprasad Chattopadhyay, 2010, 227-244.

104. Rios J L. Effects of triterpenes on the immune system, J. Ethnopharmacol, 128(1), 2010, 1-14.

105. Huang L R, Hao X J, Li Q J, Wang D P, Zhang J X, Luo H, Yang X S. 18 $\beta$ glycyrrhetinic acid derivatives possessing a trihydroxylated a ring are potent GramPositive antibacterial agents, J. Nat. Prod, 79(4), 2016, 721-731.

106. Terasawa T, Okada T, Nishino H. Antitumor-promoter activity of modified glycyrrhetinic acid derivatives, Synthesis and structure-activity relationships, Eur. J. Med. Chem, 27(7), 1992, 689-692.

107. Zhang A, Zhang X, Xia C, Xu H, Wang H. Glycyrrhetinic acid ester derivative synthesis method and deoxoglycyrrhetinic acid ester compound, US20120172438A1, 2012.

108. Zhang A, Zhang X, Xia C, Xu H, Wang H. Glycyrrhetinic acid ester derivative synthesis method and deoxoglycyrrhetinic acid ester compound, WO/2010/139253, 2010.

109. Baltina L A. Chemical modification of glycyrrhizic acid as a route to new bioactive compounds for medicine, Curr. Med. Chem, 10(2), 2003, 155-171.

110. Kondratenko R M, Baltina L A, Vasil' Eva, E V, Baltina Jr L A, Ismagilova A F, Nasyrov K M, Baschenko N Z, Kireeva R M, Fridman S M, Tolstikov G A. Synthesis and immunostimulating activity of cysteinecontaining derivatives of glycyrrhizic acid, Russ. J. Bioorganic Chem, 30(1), 2004, 6167.

111. Csuk R, Schwarz S, Siewert B, Kluge R, Strohl D. Synthesis and antitumor activity of ring A modified glycyrrhetinic acid derivatives, Eur. J. Med. Chem, 46(11), 2011, 5356-5369.

112. Nakashima $H$, Matsui $T$, Yoshida O, Isowa Y, Kido Y, Motoki Y, Ito M, Shigeta S, Mori

March - April

67 
Farid A. Badria. / International Journal of Research in Pharmaceutical and Nano Sciences. 9(2), 2020, 46-71.

T, Yamamoto N. A new anti-human immunodeficiency virus substance, glycyrrhizin sulfate, Endowment of glycyrrhizin with reverse transcriptaseinhibitory activity by chemical modification, Japanese J. Cancer Res, 78(8), 1987, 767771.

113. Wagner H, Hikino H, Farnsworth N R. Economic and medicinal plant research, Academic Press, $3^{\text {rd }}$ Edition, 2012, 150.

114. Bafna A R, Mishra S H. Immunostimulatory effect of methanol extract of Curculigo orchioides on immunosuppressed mice, $J$. Ethnopharmacol, 104(1-2), 2006, 1-4.

115. Yesilada E, Bedir E, Calis I, Takaishi Y, Ohmoto Y. Effects of triterpene saponins from Astragalus species on in vitro cytokine release, J. Ethnopharmacol, 96(1-2), 2005, 71-77.

116. Raphael T J, Kuttan G. Effect of naturally occurring triterpenoids glycyrrhizic acid, ursolic acid, oleanolic acid and nomilin on the immune system, Phytomedicine, 10(6-7), 2003, 483-489.

117. Shukla S, Bajpai V K, Kim M. Plants as potential sources of natural immunomodulators, Rev. Environ. Sci. Bio/Technology, 13(1), 2014, 17-33.

118. Abdel-Rahman S A, El-Gohary N S, ElBendary E R, El-Ashry S M and Shaaban M I. Synthesis, antimicrobial, antiquorum-sensing, antitumor and cytotoxic activities of new series of cyclopenta (hepta) [b] thiophene and fused cyclohepta [b] thiophene analogs, European Journal of Medicinal Chemistry, Corpus ID: 36852540, 140, 2017, 200-211.

119. Agarwal R K, Silver P B, Caspi R R. Rodent models of experimental autoimmune uveitis, Autoimmun. Methods Protoc, 900, 2012, 443469.

120. Baell J B and Holloway G A. New substructure filters for removal of pan assay interference compounds (PAINS) from screening libraries and for their exclusion in

Available online: www.uptodateresearchpublication.com bioassays, Journal of Medicinal Chemistry, 53(7), 2010, 2719-2740.

121. Beseda I, Czollner L, Shah P S, Khunt R, Gaware R, Kosma P, Stanetty C, Ruiz-Ruiz M C Del, Amer H, Mereiter K, Cunha T Da, Odermatt A, Claßen-Houben D, Jordis U. Synthesis of glycyrrhetinic acid derivatives for the treatment of metabolic diseases, Bioorganic Med. Chem, 18(1), 2010, 433454.

122. Bowden K, Heilbron I M, Jones E R H, Weedon B C L. 13. Researches on acetylenic compounds. Part I. The preparation of acetylenic ketones by oxidation of acetylenic carbinols and glycols, J. Chem. Soc, 1946, 3945.

123. Boyum A. Isolation of lymphocytes, granulocytes and macrophages, Scand. $J$. Immunol, 5(S5), 1976, 9-15.

124. Chand N, Eyre P. The Schultz-Dale reaction: a review, Agents Actions, 8(3), 1978, 171-184.

125. Chaturvedula V S P, Yu O, Mao G. Isolation and NMR Spectral Assignments of 18Glycyrrhetinic acid-3-O-D-glucuronide and 18-Glycyrrhetinic acid, Corpus ID: 55619469, 2014.

126. Cheng S Y, Wang C M, Cheng H L, Chen $\mathrm{H}$ J, Hsu Y M, Lin Y C, Chou C H. Biological activity of oleanane triterpene derivatives obtained by chemical derivatization, Molecules, 18(10), 2013, 13003-13019.

127. Cohen P L, Eisenberg R A. Lpr and gld: single gene models of systemic autoimmunity and lymphoproliferative disease, Annu. Rev. Immunol, 9, 1991, 243-269.

128. Colic M, Vucevic D, Kilibarda V, Radicevic N, Savic, M. Modulatory effects of garlic extracts on proliferation of T-lymphocytes in vitro stimulated with concanavalin A, Phytomedicine, 9(2), 2002, 117-124.

129. Constantinescu C S, Farooqi N, O'Brien K, Gran B. Experimental autoimmune encephalomyelitis (EAE) as a model for 
Farid A. Badria. / International Journal of Research in Pharmaceutical and Nano Sciences. 9(2), 2020, 46-71.

multiple sclerosis (MS), Br. J. Pharmacol, 164(4), 2011, 1079-1106.

130. Cooper J C, Turcasso N. Immunostimulatory effects of beta-1, 3glucan and acemannan, JANA, 2, 1999, 5-11.

131. Dey P, Chaudhuri T K. In vitro modulation of TH1 and TH2 cytokine expression by edible tuber of Dioscorea alata and study of correlation patterns of the cytokine expression, Food Sci. Hum. Wellness, 3(1), 2014, 1-8.

132. El-Desouky S, Ryu S Y and Kim Y K. A new cytotoxic acylated apigenin glucoside from Phyllanthus emblica L, Natural Product Research, 22(1), 2008, 91-95.

133. El Sayed K A, Laphookhieo S, Yousaf M, Prestridge J A, Shirode A B, Wali, V B, Sylvester P W. Semisynthetic and biotransformation studies of (1 S, 2 E, 4 S, 6 R, 7 E, 11 E)-2, 7, 11-cembratriene-4, 6-diol, J. Nat. Prod, 71(1), 2008, 117-122.

134. El-Sheikh A A K. Renal transport and drug interactions of immunosuppressants, Bulletin of the Polish Academy of Sciences Mathematics, 2008.

135. Feldman A M, McNamara D. Myocarditis, N. Engl. J. Med, 343(19), 2000, 1388-1398.

136. Gell P G H, Hinde I T. Observations on the histology of the Arthus reaction and its relation to other known types of skin hypersensitivity, Int. Arch. Allergy Immunol, 5(1), 1954, 23-46.

137. Giarratana N, Penna G, Adorini L. Animal Models of Spontaneous Autoimmune Disease, Immunol. Toler. Methods Protoc, 380, 2007, 285-311.

138. Glant T T, Mikecz K, Arzoumanian A, Poole A R. Proteoglycan-induced arthritis in balb/c mice, Arthritis Rheum, 30(2), 1987, 201-212.

139. Goldlust M B, Schreiber W F. Use of the reversed passive Arthus reaction as a test for anti-inflammatory agents, Agents Actions, 5(1), 1975, 39-47.
140. Graebin C S, Verli H, Guimaraes J A. Glycyrrhizin and glycyrrhetic acid: scaffolds to promising new pharmacologically active compounds, J. Braz. Chem. Soc, 21(9), 2010, 1595-1615.

141. Hari Y, Urwyler A, Hurni M, Yawalkar N, Dahinden $\mathrm{C}$, Wendland $\mathrm{T}$, Braathen $\mathrm{L} \mathrm{R}$, Matter L, Pichler W J. Distinct serum cytokine levels in drug-and measles-induced exanthema, Int. Arch. Allergy Immunol, 120(3), 1999, 225-229.

142. Heilbron I, Jones E R H, Sondheimer F. 129. Researches on acetylenic compounds. Part $\mathrm{XV}$, The oxidation of primary acetylenic carbinols and glycols, J. Chem. Soc, 1949, 604-607.

143. Helferich B, Schaefer W. n-Butyryl Chloride, Organic Synthesis, JB Conant, 9, 1929, 32.

144. Higo S, Shimizu A, Masuda Y, Nagasaka S, Kajimoto Y, Kanzaki G, Fukui, M, Nagahama K, Mii A, Kaneko T. Acute Graft-VersusHost Disease of the Kidney in Allogeneic Rat Bone Marrow Transplantation, PLoS One, 9(12), 2014, e115399.

145. Hussain R F, Nouri A M E, Oliver R T D. A new approach for measurement of cytotoxicity using colorimetric assay, J. Immunol. Methods, 160(1), 1993, 89-96.

146. Ignatov D V, Prokof'Ev IuI, Ipatova O M, Timofeev V P, Medvedeva N V, Misharin Ai. A simple method for preparation of 18alphaglycyrretinic acid ant its derivatives, Bioorg. Khim, 29(4), 2002, 429-433.

147. Juyal P D, Singla L D. Herbal immunomodulatory and therapeutic approaches to control parasitic infection in livestock, India Dep. Vet. Parasitol. Coll. Vet. Sci. Punjab Agric. Univ, 2001, 1-8.

148. Kikuzaki H, Tsai S M, Nakatani N. Gingerdiol related compounds from the rhizomes of Zingiber officinale, Phytochemistry, 31(5), 1992, 1783-1786. 
Farid A. Badria. / International Journal of Research in Pharmaceutical and Nano Sciences. 9(2), 2020, 46-71.

149. Kissinger R, Myl A D. Improvements to the plaque assay for antibody secreting cells, $J$. Immunol. Methods, 66(2), 1984, 377-382.

150. Kodama M, Matsumoto Y, Fujiwara M, Masani F, Izumi T, Shibata A. A novel experimental model of giant cell myocarditis induced in rats by immunization with cardiac myosin fraction, Clin. Immunol. Immunopathol, 57(2), 1990, 250-262.

151. Kong Y C. Experimental autoimmune thyroiditis in the mouse, Curr. Protoc. Immunol, 78(1), 2007, 15-17.

152. Kumar D, Arya V, Kaur R, Bhat Z A, Gupta $\mathrm{V}$ K, Kumar V. A review of immunomodulators in the Indian traditional health care system, J. Microbiol. Immunol. Infect, 45(3), 2012, 165-184.

153. Leban J, Vitt D. Human dihydroorotate dehydrogenase inhibitors, a novel approach for the treatment of autoimmune and inflammatory diseases, Arzneimittel forschung, 61(1), 2010, 66-72.

154. Lennon V A, Lindstrom J M, Seybold M E. Experimental autoimmune myasthenia: A model of myasthenia gravis in rats and guinea pigs, J. Exp. Med, 141(6), 1975, 1365-1375.

155. Li J J. Williamson ether synthesis, In: Name Reactions, Springer, $2^{\text {nd }}$ Edition, 2003, 437.

156. Luo Y, Dorf M E. Delayed-Type Hypersensitivity, Curr. Protoc. Immunol, 55(1), 2001, 1-5.

157. Makimura S, Brinkmann V, Mossmann H, Fischer H. Chemiluminescence response of peritoneal macrophages to parasitized erythrocytes and lysed erythrocytes from Plasmodium berghei-infected mice, Infect. Immun, 37(2), 1982, 800-804.

158. Mathison R, Davison J S, Befus A D. Platelet activating factor and systemic anaphylaxis in Nippostrongylus brasiliensis-sensitized rats: differential effects of PAF antagonists, Br. J. Pharmacol, 106(2), 1992, 263-266.

159. Mican J A M, Arora N, Burd P R, Metcalfe D D. Passive cutaneous anaphylaxis in mouse Available online: www.uptodateresearchpublication.com skin is associated with local accumulation of interleukin-6 mRNA and immunoreactive interleukin-6 protein, J. Allergy Clin. Immunol, 90(5), 1992, 815-824.

160. Morgan E. Vogel's textbook of practical organic chemistry, Endeavour, $5^{\text {th }}$ Edition, 1990, 1552.

161. Mosmann T. Rapid colorimetric assay for cellular growth and survival: application to proliferation and cytotoxicity assays, $J$. Immunol. Methods, 65(1-2), 1983, 55-63.

162. Munn D H, Shafizadeh E, Attwood J T, Bondarev I, Pashine A, Mellor A L. Inhibition of $\mathrm{T}$ cell proliferation by macrophage tryptophan catabolism, J. Exp. Med, 189(9), 1999, 1363-1372.

163. Ohashi N, Sugiura T, Isozaki T, Yamamoto T, Hishida A. Anti-glomerular basement membrane antibody-induced glomerulonephritis with periglomerular granulomatous reaction and massive renal eosinophilic infiltration, Am. J. Kidney Dis, 42(3), 2003, e28-35.

164. Perkins J. Fatal drug-induced immune hemolytic anemia due to cefotetan; A case study, Asian J. Transfus. Sci, 2(1), 2008, 2023.

165. Re R, Pellegrini N, Proteggente A, Pannala A, Yang M, Rice-Evans C. Antioxidant activity applying an improved ABTS radical cation decolorization assay, Free Radic. Biol. Med, 26(9-10), 1999, 1231-1237.

166. Shah B N. Textbook of pharmacognosy and phytochemistry, Elsevier, India, $1^{\text {st }}$ Edition, 2009, 586.

167. Shahlaei M, Ghanadian S M, Ayatollahi A M, Mesaik M A, Abdalla O M, Afsharypour S, Rabbani M. Molecular modeling, structure activity relationship and immunomodulatory properties of some lupeol derivatives, Med. Chem. Res, 22(4), 2013, 1795-1803.

168. Singh S, Taneja M, Majumdar D K. Biological activities of Ocimum sanctum $\mathrm{L}$. fixed oil--an overview, Indian J. Exp. Biol, 45(5), 2007, 403-412.

March - April 70 
Farid A. Badria. / International Journal of Research in Pharmaceutical and Nano Sciences. 9(2), 2020, 46-71.

169. Sommerhoff C P, Osborne M L, Lazarus S C. Effect of inhibitors on histamine release from mast cells recovered by bronchoalveolar lavage in Basenji-greyhound and mongrel dogs, Agents Actions, 31(3-4), 1990, 183-189.

170. Stahl E. Thin-Layer Chromatography - A Laboratory Handbook, Springer Int. Student $E d, 2^{\text {nd }}$ Edition, 1969, 1-23.

171. Stuart J M, Cremer M A, Townes A S, Kang A H. Type II collagen-induced arthritis in rats. Passive transfer with serum and evidence that IgG anticollagen antibodies can cause arthritis, J. Exp. Med, 155(1), 1982, 1-16.

172. Takasato F, Morita R, Schichita T, Sekiya T, Morikawa Y, Kuroda T, Niimi M, Yoshimura A. Prevention of allogeneic cardiac graft rejection by transfer of ex vivo expanded antigen-specific regulatory T-cells, PLoS One, 9(2), 2014, e87722.

173. Van Eden W, Wagenaar-Hilbers J, Wauben M H M. Adjuvant arthritis in the rat, Curr. Protoc. Immunol, 19(1), 1996, 14-15.

174. Weinfeld I, Birman E G, Paula C R. Macrophage phagocytosis of Candida albicans. An in vitro study, Rev. Odontol. da Univ. Sao Paulo, 13(3), 1999, 233-238.

175. Yu D, Sakurai Y, Chen C H, Chang F R, Huang L, Kashiwada Y, Lee K H. Anti-AIDS agents 69. Moronic acid and other triterpene derivatives as novel potent anti-HIV agents, $J$. Med. Chem, 49(18), 2006, 5462-5469.

Please cite this article in press as: Farid A. Badria. Natural products and medicinal plants: Boosting Immune System against COVID-19 and other viruses, International Journal of Research in Pharmaceutical and Nano Sciences, 9(2), 2020, 46-71. 